\title{
Improved time and frequency synchronization in presence of imperfect channel state information
}

\author{
Cong Luong Nguyen ${ }^{1}$, Anissa Mokraoui ${ }^{1}$, Pierre Duhamel ${ }^{2}$ and Nguyen Linh-Trung ${ }^{3}$
}

\begin{abstract}
This paper addresses altogether time and frequency synchronization in IEEE 802.11a orthogonal frequency division multiplexing (OFDM) wireless communication systems. The proposed algorithms have two main features: (i) they make use of an additional source of information available at IEEE 802.11 a physical layer, on top of the usual ones typically adopted for synchronization such as training sequences. This additional source of information is provided by the higher layers of the communication protocol. In fact, when the carrier sense multiple access with collision avoidance (CSMA/CA) protocol is activated, the receiver is able to predict some parts of the SIGNAL field that are classically assumed unknown. Moreover, during the negotiation of the transmission medium reservation, the exchanged frames not only help the receiver to predict the SIGNAL field but also to obtain information about the channel state. (ii) Based on this property, we propose a joint MAP time and frequency synchronization algorithm using all available information. Finally, the time synchronization is fine tuned by means of a specific metric in the frequency domain that allows us to minimize the expectation of the transmission error function over all channel estimate errors. Simulation results compliant with the IEEE 802.11a standard in both indoor and outdoor environments show that the proposed algorithm drastically improves the performance in terms of synchronization failure probability and bit error ratio, compared to state-of-the-art algorithms.
\end{abstract}

Keywords: IEEE 802.11a; CSMA/CA; RtS control frame; CtS control frame; SIGNAL field; Frequency synchronization; Time synchronization

\section{Introduction}

Due to its flexibility and efficiency in coping with interference channels, orthogonal frequency division multiplexing (OFDM) has been adopted by many recent standards, among which is the IEEE 802.11a standard [1]. However, the main disadvantage of this technique is the sensitivity to inter-symbol interference (ISI) and inter-carrier interference (ICI) caused by time and frequency synchronization errors $[2,3]$. Therefore, accurate time and frequency synchronization is absolutely required at the receiver. A synchronization process can be performed using either some redundant information (i.e., non-data-aided (NDA) techniques) or training sequences (i.e., data-aided (DA) techniques) included in the transmitted physical packet.

\footnotetext{
*Correspondence: anissa.mokraoui@univ-paris13.fr

1 L2TI, Institut Galilée, Université Paris 13, Sorbonne Paris Cité, 99 avenue Jean-Baptiste Clément, F93430 Villetaneuse, France

Full list of author information is available at the end of the article
}

The NDA approach usually employs the extension of an OFDM symbol, known as the cyclic prefix (CP), which corresponds to the last OFDM samples duplicated in front of the OFDM symbol. The correlation property between the CP and its copy in the OFDM symbol has been exploited for time synchronization [4-6], ([7], p.163), frequency synchronization ([7], p.170),[8], or both time and frequency synchronization [9-12]. A maximum likelihood (ML) criterion is commonly used. The symbol timing and the carrier frequency offset (CFO) parameters are estimated by searching the index maximizing the ML function. NDA techniques are characterized by bandwidth and transmission power savings since no additional information is requested (such as training sequences). However, they are not very robust to multipath distortion and most of them face a significant performance deterioration over frequency-selective fading channels [13]. Moreover, due to the distance between the $\mathrm{CP}$ and its copy, the range of

\section{Springer}

(c) 2015 Nguyen et al.: licensee Springer. This is an Open Access article distributed under the terms of the Creative Commons Attribution License (http://creativecommons.org/licenses/by/4.0), which permits unrestricted use, distribution, and reproduction in any medium, provided the original work is properly credited. 
normalized CFO estimation is limited within $[-0.5,+0.5]$ ([7], p.170).

The DA approach exploits training sequences which are either designed specifically or specified by some standards. Schmidl and Cox in [14] proposed to use a training sequence that is composed of two symbols for time and frequency synchronization. The first symbol consists of two identical halves. The receiver applies an autocorrelation function (ACF) on the received signal, and the maximum absolute value of this function is chosen as an estimate of the symbol timing. At the estimated position, the CFO estimation is then performed in two main steps. The first step estimates the fractional frequency offset (FFO) based on the ACF phase between the two halves of the first symbol. The integer frequency offset (IFO) is estimated according to a cross-correlation function (CCF) computed between the known second symbol and the received symbol in the frequency domain. The authors of [15] proposed to reduce the length of the training sequence by making use of a single additional training sequence generated according to the first data symbol. The symbol timing and CFO estimates are obtained via the ACF calculated between the two identical symbols. In [16], a joint CFO and channel estimation using the maximum a posteriori (MAP) criterion is developed. Specifically, a posteriori probability function of the CFO and channel coefficients is built. The unknown parameters are then estimated as the values maximizing this function. In [17], a ML time synchronization offset (TSO) and CFO estimation method for OFDM systems with ten periodic preambles is developed. However the proposed solution is not completely adapted to the 802.11a standard. Indeed the two LTF repetitions, reserved for channel estimation and fine frequency synchronization, are not considered in the paper. In [18], the time and frequency synchronization algorithm is adapted to the structure of the IEEE 802.11a PREAMBLE, which is composed of a short training field (STF) and a long training field (LTF). Coarse time synchronization (CTS) and coarse frequency synchronization (CFS) are based on the ACF of the STF. Fine frequency synchronization (FFS) and fine time synchronization (FTS) employ the ACF of the LTF. However, in [19], the FTS is performed with the CCF between the received signal and a part of the LTF. In [20], the FTS is performed jointly with channel estimation. A set of possible time offsets is given; and for each value of the set, the channel impulse response (CIR) is estimated using the least square (LS) criterion. The estimated CIR allows the identification of the beginning of the LTF symbols. The estimated time offset is the one that minimizes the mean square error (MSE) criterion between the known and the estimated LTF symbols.

Based on the respective advantages of both NDA and DA approaches, new algorithms jointly exploiting them have been developed for time synchronization in [21-23] and for both time and frequency synchronization in [24], all for the IEEE 802.11a wireless system. This is made feasible by a careful study of the communication protocol so as to artificially increase the size of the training sequences and to characterize the channel estimates. To improve the performance of the algorithm in [24], we propose in this paper a multistage time and frequency synchronization algorithm in the presence of imperfect channel state information. In fact, the analysis of the results provided in [2022 ] shows that the strategy of channel estimation strongly impacts the accuracy of symbol timing estimation. Indeed, reducing the channel estimation error improves the time synchronization performance. Rather than trying to find the best channel estimator, we derive a timing metric that minimizes the expectation of the transmission error probability over all channel estimation errors. This strategy is carried out in the frequency domain when the CFO of the received signal is assumed to be perfectly compensated by the proposed algorithm.

This paper is organized as follows. The next section introduces the IEEE 802.11a wireless communication system. Section 3 reviews some important synchronization algorithms. Section 4 describes the proposed time and frequency synchronization algorithm. Section 5 discusses the simulation results. Section 6 concludes the work.

\section{Context - IEEE 802.11 a communication system}

Before presenting the IEEE 802.11a wireless communication system, this section first provides a brief description of the physical packet structure. It consists of three main fields: the PREAMBLE training field, the SIGNAL field, and the DATA field (see Figure 1). The PREAMBLE field helps the mobile receiver to synchronize with the mobile transmitter. This field is composed of: (i) ten identical and known STF sequences which are used for automation gain control (AGC), diversity selection, signal detection, and CFS; and (ii) two identical and known LTF sequences which are reserved for channel estimation and FFS. The SIGNAL field provides information about the transmission rate (in Mbits/s) and the length of the DATA field (in octets).

Before being transmitted, the IEEE 802.11a physical packet must follow the various processing steps summarized in Figure 2. The binary SIGNAL sequence is used as input to the convolutional encoder with a constraint length $K=7$, a polynomial generator [171,133], and a code rate $R=1 / 2$. Its outputs are interleaved by a known interleaver and then binary phase shift keying (BPSK) modulated. This is followed by a pilot insertion and the OFDM modulation, with an $N$-point inverse fast Fourier transform (IFFT). DATA field information bits are applied to a scrambler before the convolutional encoder. Moreover, variable code rates (with $R=1 / 2,2 / 3$, 


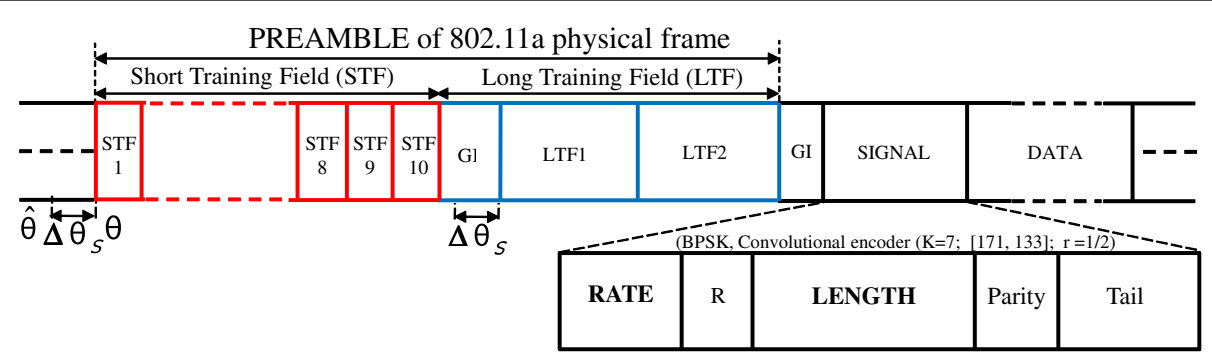

Figure 1 IEEE 802.11a physical packet.

or 3/4) and different modulations (e.g., M-PSK or MQAM) are available, depending on the desired data rate to achieve.

The IFFT is applied to the symbols $X(k)(0 \leq k \leq N-1)$ to get the time domain samples $x(n)$. These samples constitute the OFDM symbol, which is preceded by a CP. The samples $x(n)$ are then shaped by a specific window before being transmitted via a multipath fading channel, described by a finite impulse response (FIR) filter of length $L$. At the receiver, the discrete baseband signal $r_{\Delta}(n)$ is expressed as

$$
r_{\Delta}(n)=\sum_{i=0}^{L-1} h(i) x(n-i-\theta) e^{j \frac{2 \pi \epsilon(n-\theta)}{N}}+g(n),
$$

where $h(i)$ is the slowly time-varying discrete complex CIR with $\sum_{i=0}^{L-1} \mathrm{E}\left\{|h(i)|^{2}\right\}=1$ ( $\mathrm{E}$ is the expectation operator); $L$ is the number of channel taps, $g(n)$ is the complex AWGN samples with variance $\sigma_{g}^{2}, \theta$ is the symbol timing, and $\epsilon=\Delta F_{c} T$ is the normalized CFO, with $\Delta F_{c}$ being the carrier frequency offset between the transmitter and the receiver and $T$ being the OFDM symbol duration. Estimating $\theta$ and $\epsilon$ is the objective of the following sections.

\section{State of the art on time and frequency synchronization}

This section briefly reviews some important synchronization algorithms. We focus on the algorithm developed in [16] since it will be modified later to fit the IEEE 80211a standard.

Synchronization algorithms can be classified in two categories. One is related to redundant information (NDA) (see, e.g., [4,6]) and the other to training sequences (DA) (see, e.g., [16,18,19]). In [6], the OFDM CP symbol is exploited. The symbol timing $\theta$ is estimated by searching the index that gives the minimum difference between two sliding windows and is given by

$$
\widehat{\theta}=\arg \min _{\theta} \sum_{n=0}^{N_{g}-1}|r(n+\theta)-r(n+\theta+N)|,
$$

where $r(n)$ is the received signal, $N_{g}$ is the length of the $\mathrm{CP}$, and $N$ is the length of the OFDM symbol. However if a CFO exists, this solution may be inaccurate. To deal with this situation, the authors of [4] proposed to minimize the squared difference between the received signal, corresponding to the first sliding window, and the conjugate received signal, associated with the second slid-

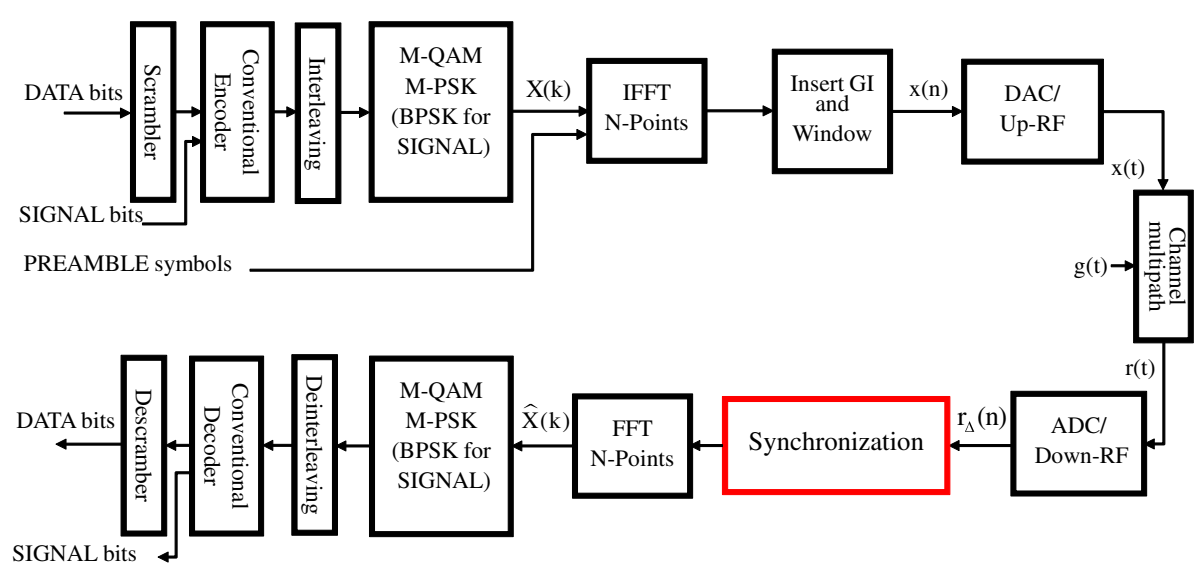

Figure 2 Wireless communication system using OFDM. 
ing window. The estimated symbol timing is then given by

$$
\widehat{\theta}=\arg \min _{\theta} \sum_{n=0}^{N_{g}-1}\left(|r(n+\theta)|-\left|r^{*}(n+\theta+N)\right|\right)^{2} .
$$

More classically, the symbol timing can also be estimated as the index which maximizes the ACF by the following:

$$
\widehat{\theta}=\arg \max _{\theta}\left|\sum_{n=0}^{N_{g}-1} r(n+\theta) r^{*}(n+\theta+N)\right| .
$$

In $[18,19]$, the proposed DA algorithms have been adapted to the IEEE 802.11a standard. To estimate the symbol timing, these algorithms proceed in two main stages: (i) CTS, then (ii) FTS. The CTS stage exploits the STF (i.e., 160 samples) to estimate the symbol timing as

$$
\widehat{\theta}=\arg \max _{\theta} \frac{\left|\sum_{n=0}^{143} r^{*}(n+\theta) r(n+\theta+16)\right|}{\sum_{n=0}^{143}|r(n+\theta)|^{2}} .
$$

The coarse CFO is then estimated by

$$
\widehat{\epsilon}_{\mathrm{c}}=\frac{N}{2 \pi 16} \arg \left\{\sum_{n=0}^{143} r^{*}(n+\hat{\theta}) r(n+\hat{\theta}+16)\right\},
$$

where $\arg \{\cdot\}$ denotes the argument of a complex number. To define $\widehat{\epsilon}_{\mathrm{c}}$, the ACF of the LTF repetitions is computed to obtain

$\widehat{\epsilon}_{\mathrm{f}}=\frac{N}{2 \pi 64} \arg \left\{\sum_{n=0}^{63} r^{*}(n+\hat{\theta}+192) r(n+\hat{\theta}+256)\right\}$.

Thus, the received signal is compensated by the following frequency offset:

$$
\hat{\epsilon}=\widehat{\epsilon}_{\mathrm{c}}+\widehat{\epsilon}_{\mathrm{f}}
$$

Then, by computing the CCF between the compensated signal, $r^{\prime}(n)$, and a part of the known LTF, $g_{\mathrm{LTF}}(n)$ (the first 32 samples over the 128 samples of the LTF), the remaining time offset is estimated as the one, in the possible predefined positions of the set $\boldsymbol{\Lambda}$, at which the CCF achieves the maximum and is thus given by

$$
\Delta \widehat{\theta}=\arg \max _{\Delta \theta \in \boldsymbol{\Lambda}}\left|\sum_{n=0}^{31} g_{\mathrm{LTF}}^{*}(n) r^{\prime}(n+\Delta \theta)\right|^{2} .
$$

To improve the accuracy of CFO estimation, a joint MAP channel estimation and frequency synchroniza- tion is developed in [16]. A transmitted burst consisting of some OFDM pilot symbols is exploited by the receiver. Note that this solution requires the symbol timing knowledge to perfectly compensate the time offset of the received signal. For convenience, the received signal corresponding to one OFDM pilot symbol of length $N$ is expressed in matrix form as follows:

$$
\mathbf{r}^{\mathrm{p}}=\boldsymbol{\Phi}_{\epsilon}^{\mathrm{p}} \mathbf{S}^{\mathrm{p}} \mathbf{h}+\mathbf{g}
$$

where

$\mathbf{r}^{\mathrm{p}}=[r(n), r(n+1), \ldots, r(n+N-1)]^{T} ;$

$\boldsymbol{\Phi}_{\epsilon}^{\mathrm{P}}=\operatorname{diag}\left\{e^{j 2 \pi \epsilon n / N}, e^{j 2 \pi \epsilon(n+1) / N}, \ldots, e^{j 2 \pi \epsilon(n+N-1) / N}\right\} ;$

$\mathbf{S}^{\mathrm{p}}=\left[\mathbf{S}_{0}^{\mathrm{p}}, \mathbf{S}_{1}^{\mathrm{p}}, \ldots, \mathbf{S}_{L-1}^{\mathrm{p}}\right]$

$\mathbf{S}_{l}^{\mathrm{p}}=[x(n-l), x(n+1-l), \ldots, x(n+N-1-l)]^{T}$ with

$l=0, \ldots, L-1$;

$\mathbf{h}=[h(0), h(1), \ldots, h(L-1)]^{T} ;$

$\mathbf{g}=[g(n), g(n+1), \ldots, g(n+N-1)]^{T}$.

The superscript 'p' indicates 'pilot,' $r(n)$ is given by Equation 1 (with $\theta=0$ ), and $x(n)$ is the known pilot sample in time domain. The MAP estimates of the channel coefficients and the normalized CFO are given by

$$
\{\hat{\mathbf{h}}, \hat{\epsilon}\}=\arg \max _{\mathbf{h}, \epsilon} \ln P\left(\mathbf{h}, \epsilon \mid \mathbf{r}^{\mathrm{p}}\right),
$$

where $P(\cdot)$ is the a posteriori joint probability density function (pdf) of $\mathbf{h}$ and $\epsilon$, given $\mathbf{r}^{\mathrm{P}}$. Under the assumption that $\epsilon$ is uniformly distributed over $\left[-\epsilon_{0}, \epsilon_{0}\right]$, we have

$$
\{\hat{\mathbf{h}}, \hat{\epsilon}\}=\arg \min _{\mathbf{h}, \epsilon} f_{\mathrm{MAP}}(\mathbf{h}, \epsilon),
$$

with

$$
f_{\mathrm{MAP}}(\mathbf{h}, \epsilon)=\frac{1}{\sigma_{g}^{2}}\left\|\mathbf{r}^{\mathrm{p}}-\boldsymbol{\Phi}_{\epsilon}^{\mathrm{p}} \mathbf{S}^{\mathrm{p}} \mathbf{h}\right\|^{2}+\mathbf{h}^{H} \mathbf{R}_{\mathbf{h}}^{-1} \mathbf{h},
$$

where $\mathbf{R}_{\mathbf{h}}$ is the channel covariance matrix. The gradient vector of $f_{\mathrm{MAP}}(\mathbf{h}, \epsilon)$, with respect to $\mathbf{h}^{H}$, is set to zero in order to produce the following MAP channel estimate:

$$
\widehat{\mathbf{h}}=\left[\left(\mathbf{S}^{\mathrm{p}}\right)^{H} \mathbf{S}^{\mathrm{p}}+\sigma_{g}^{2} \mathbf{R}_{\mathbf{h}}^{-1}\right]^{-1}\left(\mathbf{S}^{\mathrm{p}}\right)^{H}\left(\boldsymbol{\Phi}_{\epsilon}^{\mathrm{p}}\right)^{H} \mathbf{r}^{\mathrm{p}} .
$$

Substituting Equation 13 into $f_{\mathrm{MAP}}(\mathbf{h}, \epsilon)$ gives the CFO estimate

$$
\hat{\epsilon}=\arg \min _{\epsilon} g_{\mathrm{MAP}}(\epsilon)
$$


where

$$
g_{\mathrm{MAP}}(\epsilon)=\left(\mathbf{r}^{\mathrm{p}}\right)^{H} \boldsymbol{\Phi}_{\epsilon}^{\mathrm{p}}\left(\mathbf{S}^{\mathrm{p}}\right)^{+}\left(\boldsymbol{\Phi}_{\epsilon}^{\mathrm{p}}\right)^{H} \mathbf{r}^{\mathrm{p}},
$$

with

$$
\left(\mathbf{S}^{\mathrm{p}}\right)^{+}=\mathbf{S}^{\mathrm{p}}\left[\left(\mathbf{S}^{\mathrm{p}}\right)^{H} \mathbf{S}^{\mathrm{p}}+\mathbf{R}_{\mathbf{h}}^{-1} \sigma_{g}^{2}\right]^{-1}\left(\mathbf{S}^{\mathrm{p}}\right)^{H} .
$$

To solve Equation 14, the Newton-Raphson iteration method is applied

$$
\hat{\epsilon}_{i+1}=\hat{\epsilon}_{i}-\left.\left[\frac{\partial^{2} g(\epsilon)}{\partial \epsilon^{2}}\right]^{-1} \frac{\partial g(\epsilon)}{\partial \epsilon}\right|_{\hat{\epsilon}=\epsilon_{i}}
$$

where $\hat{\epsilon}_{i}$ represents the CFO estimate at the $i$ th iteration, and

$$
\begin{aligned}
\frac{\partial g(\epsilon)}{\partial \epsilon}= & 2 \Re\left\{\left(\mathbf{r}^{\mathrm{p}}\right)^{H} \mathbf{Q}^{\mathrm{p}} \boldsymbol{\Phi}_{\epsilon}^{\mathrm{p}}\left(\mathbf{S}^{\mathrm{p}}\right)^{+}\left(\boldsymbol{\Phi}_{\epsilon}^{\mathrm{p}}\right)^{H} \mathbf{r}^{\mathrm{p}}\right\}, \\
\frac{\partial g^{2}(\epsilon)}{\partial \epsilon^{2}}= & 2 \Re\left\{\left(\mathbf{r}^{\mathrm{p}}\right)^{H}\left(\mathbf{Q}^{\mathrm{p}}\right)^{2} \boldsymbol{\Phi}_{\epsilon}^{\mathrm{p}}\left(\mathbf{S}^{\mathrm{p}}\right)^{+}\left(\boldsymbol{\Phi}_{\epsilon}^{\mathrm{p}}\right)^{H} \mathbf{r}^{\mathrm{p}}+\right. \\
& \left.\left.+\left(\mathbf{r}^{\mathrm{p}}\right)^{H} \mathbf{Q}^{\mathrm{p}} \boldsymbol{\Phi}_{\epsilon}^{\mathrm{p}}\left(\mathbf{S}^{\mathrm{p}}\right)^{+}\left(\mathbf{Q}^{\mathrm{p}}\right)^{H}\left(\boldsymbol{\Phi}_{\epsilon}^{\mathrm{p}}\right)^{H} \mathbf{r}^{\mathrm{p}}\right)\right\}, \\
\mathbf{Q}^{\mathrm{p}}= & j \frac{2 \pi}{N} \operatorname{diag}\{n, n+1, \ldots, n+N-1\} .
\end{aligned}
$$

\section{Proposed multistage DA-NDA time and frequency synchronization algorithm}

The proposed multistage DA-NDA synchronization algorithm not only addresses the problem of estimating the symbol timing, $\theta$ but also the CFO, $\varepsilon$. The algorithm is composed of an initialization stage (see Section 4.1) followed by three main stages as summarized in Figure 3: (i) the CTS stage, which uses not only STF but also the predicted SIGNAL field and channel estimate according to the exchanged request to send (RtS) control frame (see Section 4.2); (ii) the joint MAP frequency and time synchronization stage (see Section 4.3); and (iii) the frequency domain-based fine timing estimation stage (see Section 4.4). In what follows, we present each of the four stages.

\subsection{Initialization stage: extraction of new information source to be exploited at physical layer packet}

Assume that the CSMA/CA mechanism is triggered to avoid collisions between mobile stations in the same network thereby using request to send/clear to send (RtS/CtS) control frames. To exploit the information carried by these control frames, the initialization stage first ensures that the stations are synchronized during the negotiation of the transmission medium reservation and then extracts new information source to be exploited by the receiver in addition to the conventional training sequences specified by the $802.11 \mathrm{n}$ standard.

Since the RtS/CtS control frames are sent with higher power levels than the nominal transmission power level of the DATA frames to guarantee that all stations in the same network should hear these control sequences (see [25]), we use the synchronization algorithm developed in [19] to estimate the symbol timing and CFO parameters based on the specified training sequences of the frames. Indeed, this algorithm provides reasonable performance for relatively higher signal to noise ratio compared to DATA frames. For symbol timing estimation, this algorithm is based on two parts. The first part, called coarse time synchronization, is an adaptation of the ACF method proposed by Schmidl and Cox [14]. The second part, called fine time synchronization, is then carried out using a CCF between the received signal and a part of the long training symbol. Moreover, this algorithm can work with the maximum $\mathrm{CFO} \epsilon$ allowed by the standard.

Before sending any DATA information, the transmitter initiates the communication by sending a RtS control frame to ask the receiver if it is available [26] (see Figure 4). Based on Equation 1, the discrete baseband signal $r_{R t S}(n)$ at the receiver is expressed as

$$
r_{\mathrm{RtS}}(n)=\sum_{i=0}^{L-1} h(i) x\left(n-i-\theta_{\mathrm{RtS}}\right) e^{j \frac{2 \pi \epsilon_{\mathrm{RtS}}\left(n-\theta_{\mathrm{RtS}}\right)}{N}}+g(n),
$$

where $\theta_{\mathrm{RtS}}$ is the symbol timing and $\epsilon_{\mathrm{RtS}}$ represents the normalized CFO. These parameters are estimated according to [19]. If the stations have been synchronized, then the exchange control information can continue. The receiver, if it is available, performs a rate adaptation algorithm by measuring the SNR level of the received RtS frame to estimate the channel conditions [27]. Then, it replies to the transmitter with a $\mathrm{CtS}$ control frame repre-

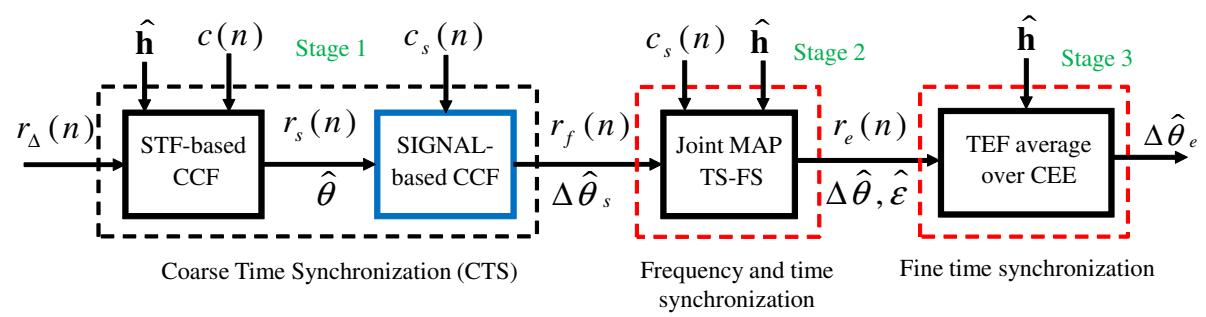

Figure 3 Proposed time and frequency synchronization algorithm. 


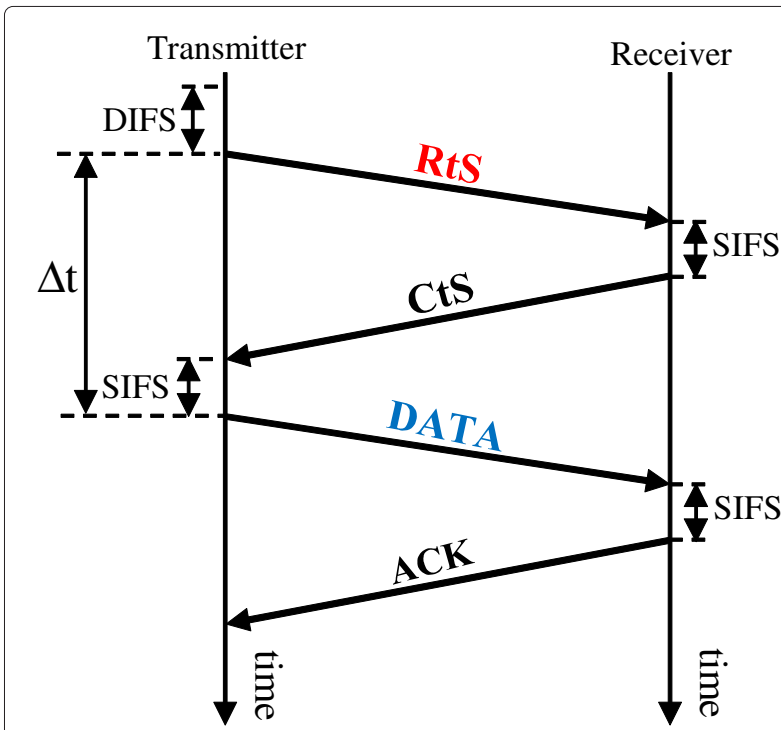

Figure $4 \mathrm{RtS} / \mathrm{CtS}$ handshake with active CSMA/CA mechanism.

sented by its discrete baseband signal $r_{\mathrm{CtS}}(n)$ expressed as

$$
\begin{aligned}
r_{\mathrm{CtS}}(n)= & \sum_{i=0}^{L-1} h(i) x\left(n-i-\theta_{\mathrm{CtS}}\right) e^{j \frac{2 \pi \epsilon_{\mathrm{CtS}}\left(n-\theta_{\mathrm{CtS}}\right)}{N}} \\
& +g(n),
\end{aligned}
$$

where $\theta_{\mathrm{CtS}}$ is the symbol timing and $\epsilon_{\mathrm{CtS}}$ represents the normalized CFO. These parameters are estimated according to [19]. If the stations have been synchronized, the $\mathrm{CtS}$ control frame aims to: (i) inform other stations (in the same network) of the unavailability of the receiver to receive information from other stations during a specified duration and (ii) suggest to the transmitter a transmission rate that should be used to transmit the physical data packet. The transmitter is then ready to send the DATA frame to the receiver, and a synchronization step is required. Therefore, the receiver predicts the SIGNAL field of the physical packet, composed of two subfields 'RATE' and 'LENGTH' (see Figure 1), which will be considered as an additional source of information for the synchronization process. Indeed, a careful study of the standard leads to the conclusion that these subfields can be known at the receiver based on the knowledge provided by the CSMA/CA protocol when it is activated as discussed below. Thereby, the receiver has a knowledge of the transmission rate concerning the 'RATE' subfield value of the SIGNAL field. The unknown 'LENGTH' subfield of the SIGNAL is then deduced from the following relationship [1]:

$$
\begin{aligned}
\text { LENGTH }= & \text { RATE } \\
& \times \frac{\left(T_{\text {packet }}-T_{\text {pre }}-T_{\text {SIGNAL }}-\left(T_{\text {symb }} / 2\right)\right)-22}{8},
\end{aligned}
$$

since the 'RATE' and the durations (in $\mu \mathrm{s}$ ) of the PREAMBLE ( $\left.T_{\text {pre }}\right)$, the SIGNAL field ( $\left.T_{\text {SIGNAL }}\right)$, and the OFDM symbol $\left(T_{\text {symb }}\right)$ are known values provided in [1]. However, the duration $T_{\text {packet }}$ (in $\mu \mathrm{s}$ ) which is required to transmit the DATA physical packet is unknown. We deduce this value from the 'DURATION' field value, extracted from the exchanged $\mathrm{CtS}$ control frame, as follows [28]:

$$
T_{\text {packet }}=\text { DURATION }-2 T_{\text {SIFS }}-T_{\mathrm{RtS}}-T_{\mathrm{ACK}},
$$

where $T_{\text {SIFS }}$ is the known duration (in $\mu \mathrm{s}$ ) of a short interframe space and $T_{\mathrm{RtS}}$ and $T_{\mathrm{ACK}}$ are respectively the known durations required to transmit RtS and acknowledgement (ACK) frames. The 'Parity' field is then deduced from the 'RATE,' 'LENGTH,' and 'R' (reserved) known values and is followed by six zero tail bits to complete the SIGNAL field. This field is now fully available to the receiver and can be exploited as an additional source of information for packet synchronization.

\subsection{First stage: coarse time synchronization}

This first stage provides a coarse estimate of the symbol timing $\theta$ of the received signal, using not only the known STF as recommended by the 802.11 a standard but also the predicted SIGNAL field. For this, the following two steps are performed.

\subsubsection{Coarse symbol timing estimation using the known STF}

To roughly estimate the time offset, the receiver generally performs a CCF between the received signal $r_{\Delta}(n)$, given by Equation 1, and the known STF sequence $c(n)$ :

$$
\hat{\theta}=\arg \max _{\theta}\left|\sum_{n=0}^{L_{\mathrm{STF}}-1} c^{*}(n) r_{\Delta}(n+\theta)\right|,
$$

where $L_{\mathrm{STF}}$ is the length of $c(n)$. However, if the transmitted signal is heavily distorted by the wireless channel, the symbol timing estimation will be affected. Therefore, to enhance the performance of this estimation, we propose to replace $r_{\Delta}(n)$ in Equation 22 by an estimate $\hat{x}(n)$ of the transmitted signal $x(n)$ that would be closer to $c(n)$. The symbol timing is then determined by

$$
\hat{\theta}=\arg \max _{\theta}\left|\sum_{n=0}^{L_{\mathrm{STF}}-1} c^{*}(n) \hat{x}(n+\theta)\right| .
$$

The estimate $\hat{x}(n)$ can be obtained during the negotiation of the transmission medium reservation managed by the CSMA/CA mechanism. Note that the RtS 
and $\mathrm{CtS}$ control frames are sent with higher power levels than the nominal transmission power level of the DATA frames in order to ensure that all stations in the same network should hear these control sequences [25]. Therefore, during the negotiation of the transmission medium reservation (i.e., $\mathrm{RtS} / \mathrm{CtS}$ ), the transmitter and the receiver are assumed to be correctly synchronized to permit the physical DATA packet transmission. Moreover, the channel is assumed to be static between the transmission duration of the RtS and DATA frames (see Figure 4) since the interval time between the transmitted physical packet and the RtS control frame (in the worst case of communication) is small (e.g., $T_{\mathrm{RtS}}+$ $T_{\mathrm{CtS}}+2 T_{\mathrm{SIFS}}=124 \mu \mathrm{s}$ with the lowest rate of $6 \mathrm{Mb} / \mathrm{s}$ ), meaning that the Doppler frequency can be considered as negligible.

Obviously, since our method relies on an estimate $\hat{x}(n)$ of the transmitted signal, this first requires some channel estimation. Note that all the control (RtS, CtS) and DATA frames use the same PREAMBLE field (see Figure 1). As mentioned in the standard for the physical packet, we base the estimation of the channel on the LTF of the RtS PREAMBLE. A MAP-based channel estimation can be obtained as

$$
\widehat{\mathbf{h}}=\left(\mathbf{Q}^{H} \mathbf{Q}+\sigma_{g}^{2} \mathbf{R}_{\mathbf{h}}^{-\mathbf{1}}\right)^{-1}\left(\mathbf{Q}^{H} \mathbf{r}_{\mathrm{RtS}}+\sigma_{g}^{2} \mathbf{R}_{\mathbf{h}}^{-\mathbf{1}} \boldsymbol{\mu}_{h}\right)
$$

where $\mathbf{r}_{\mathrm{RtS}}$ is the received vector of length $N$ corresponding to the LTF of the RtS frame signal (here, $N$ is also the length of one LTF repetition), $\mathbf{Q}=\mathbf{F}^{H} \mathbf{X F}$ where $\mathbf{F}$ is the FFT matrix of size $N \times N$ and $\mathbf{X}$ is the diagonal matrix of size $N \times N$ whose diagonal elements are the known LTF symbols, the notation $(\cdot)^{H}$ denotes the conjugate transpose operator, $\mathbf{R}_{\mathbf{h}}$ is the covariance matrix of the true channel, and $\boldsymbol{\mu}_{h}$ is the mean vector of the true channel assumed to follow a Gaussian distribution.

A precise computation of Equation 24 would require the knowledge of the power delay profile (PDP) to calculate $\mathbf{R}_{\mathbf{h}}=E\left\{\mathbf{h} \mathbf{h}^{H}\right\}$. Since this knowledge can hardly be available at the receiver, we propose to replace $\mathbf{R}_{\mathbf{h}}$ by an approximation. Under this approximation, the channel taps are independent (hence, all non-diagonal elements of $\mathbf{R}_{\mathbf{h}}$ are assumed to be zero), and the diagonal elements should contain the squared norm of the taps. These are obtained via an LS estimate of the channel $\tilde{\mathbf{h}}$ given by the IFFT of $\tilde{\mathbf{H}}=\mathbf{X}^{-1} \mathbf{r}_{\mathrm{RtS}}$, where $\mathbf{X}$ is the diagonal matrix whose elements are the known LTF symbols and $\mathbf{r}_{\mathrm{RtS}}$ is the received symbol vector.

Let $\hat{H}(k)$ (with $0 \leq k \leq N-1$ ) be the channel estimate and $R_{\Delta}(k)$ be the received symbols corresponding to the DATA frame signal in frequency domain, then the trans- mitted symbol estimate, $\hat{X}(k)$, according to a zero-forcing (ZF) equalizer, is provided by

$$
\hat{X}(k)=\frac{R_{\Delta}(k)}{\hat{H}(k)}
$$

which is then transformed to the time domain for its usage in Equation 23.

\subsubsection{Improving coarse symbol timing estimation using the predicted SIGNAL field}

The received signal is still affected by some remaining time offset (i.e., $\Delta \theta_{s}=\hat{\theta}-\theta$ ) and can be expressed as

$r_{s}(n)=\sum_{i=0}^{L-1} h(i) x\left(n-i-\Delta \theta_{s}\right) e^{j 2 \pi \epsilon\left(n-\Delta \theta_{s}\right) / N}+g(n)$.

To estimate $\Delta \theta_{s}$, as in [21,22], we exploit the 802.11a SIGNAL field as an additional training sequence at the receiver since all parts of this field are completely known (see Section 4.1). A CCF is performed between the received signal $r_{s}(n)$ and the known SIGNAL field $c_{s}(n)$ of length $L_{\text {SIG }}$ (i.e., the sum of the CP length and the SIGNAL length). The remaining time offset corresponds to the index among the set of possible values $\Theta=\left\{\Delta \theta_{s}^{(k)} \mid k=-\right.$ $K, \ldots, K ; K \in \mathbf{N}\}$ which maximizes the CCF absolute value as follows:

$$
\Delta \widehat{\theta_{s}}=\arg \max _{\Delta \theta_{s}^{(k)} \in \Theta}\left|\sum_{n=0}^{L_{\mathrm{SIG}}-1} c_{s}^{*}(n) r_{s}\left(n+\Delta \theta_{s}^{(k)}\right)\right| .
$$

After this step, the received signal becomes

$$
r_{f}(n)=\sum_{i=0}^{L-1} h(i) x(n-i-\Delta \theta) e^{j \frac{2 \pi \epsilon(n-\Delta \theta)}{N}}+g(n),
$$

where $\Delta \theta=\Delta \widehat{\theta_{s}}-\Delta \theta_{s}$ is the possible remaining time offset.

\subsection{Second stage: joint MAP time and frequency synchronization}

This section estimates the possible remaining time offset $\Delta \theta$ and the normalized frequency offset $\epsilon$ in Equation 28 . In order to do so, a joint MAP time and frequency synchronization algorithm is developed. The method is based on the frequency synchronization algorithm developed in [16] (see Section 3) which is adapted below to the IEEE 802.11a specifications and modified to take into account the fact that the time offset is not perfectly compensated. The knowledge about the SIGNAL field is also useful in this stage.

The received signal $\mathbf{r}$ corresponding to the two LTF repetitions and the SIGNAL field is expressed in a matrix form as follows:

$$
\mathbf{r}=\boldsymbol{\Phi}_{\Delta \theta, \epsilon} \mathbf{S}_{\Delta \theta} \mathbf{h}+\mathbf{g},
$$


where

$$
\begin{aligned}
\mathbf{r}= & {\left[r_{f}(n), \ldots, r_{f}\left(n+2 N+N_{G}+N_{S}-1\right)\right]^{T} } \\
\mathbf{S}_{\Delta \theta}= & {\left[\mathbf{S}_{0, \Delta \theta}, \mathbf{S}_{1, \Delta \theta}, \ldots, \mathbf{S}_{L-1, \Delta \theta}\right] } \\
\mathbf{S}_{l, \Delta \theta}= & {\left[x(n-l-\Delta \theta), \ldots, x\left(n+2 N+N_{G}+N_{S}-1\right.\right.} \\
& -l-\Delta \theta)]^{T} \\
\mathbf{h}= & {[h(0), h(1), \ldots, h(L-1)]^{T} } \\
\mathbf{g}= & {\left[g(n-\Delta \theta), \ldots, g\left(n-\Delta \theta+2 N+N_{S}+N_{G}-1\right)\right]^{T} } \\
\Phi_{\Delta \theta, \epsilon}= & \operatorname{diag}\left\{e^{j 2 \pi \epsilon(n-\Delta \theta) / N}, \ldots,\right. \\
& \left.e^{j 2 \pi \epsilon\left(n-\Delta \theta+2 N+N_{S}+N_{G}-1\right) / N}\right\},
\end{aligned}
$$

with $x(n)$ being the known LTF and SIGNAL samples in the time domain, $N$ the number of samples of one LTF repetition, $N_{S}$ the SIGNAL field length, and $N_{G}$ the GI length. The vectors $\mathbf{h}$ and $\mathbf{g}$ correspond to the CIR and the noise, respectively. The unknown parameters $\Delta \theta, \epsilon$, and $\mathbf{h}$ are jointly estimated according to the MAP criterion as follows:

$$
\{\hat{\mathbf{h}}, \Delta \widehat{\theta}, \hat{\epsilon}\}=\arg \max _{\mathbf{h}, \Delta \theta, \epsilon} \ln P(\mathbf{h}, \Delta \theta, \epsilon \mid \mathbf{r}),
$$

where $P(\cdot)$ is the a posteriori $\operatorname{pdf}$ of $\mathbf{h}, \Delta \theta$ and $\epsilon$ given $\mathbf{r}$. Note that $\epsilon$ is also assumed to be uniformly distributed in the range $\left[-\epsilon_{0}, \epsilon_{0}\right]$.

To solve this equation, a set $\boldsymbol{\Lambda}=\left\{-\Delta \theta_{M}, \ldots, \Delta \theta_{M}\right\}$, containing $2 M+1$ possible time offset values, is defined where $M$ is selected according to the maximum time deviation to cover all possible time offsets from the first stage. For a given value $\Delta \theta_{m} \in \Lambda$, the MAP-based estimated channel coefficients and the CFO are

$$
\left\{\hat{\mathbf{h}}_{\Delta \theta_{m}}, \hat{\epsilon}_{\Delta \theta_{m}}\right\}=\arg \min _{\mathbf{h}, \epsilon} f_{\mathrm{MAP}}^{(m)}(\mathbf{h}, \epsilon)
$$

where $\mathbf{r}_{\Delta \theta_{m}}$ is the received signal with the offset value $\Delta \theta_{m}$ and

$$
f_{\mathrm{MAP}}^{(m)}(\mathbf{h}, \epsilon)=\frac{1}{\sigma_{g}^{2}}\left\|\mathbf{r}_{\Delta \theta_{m}}-\boldsymbol{\Phi}_{\Delta \theta_{m}, \epsilon} \mathbf{S}_{\Delta \theta_{m}} \mathbf{h}\right\|^{2}+\mathbf{h}^{\mathbf{H}} \mathbf{R}_{\mathbf{h}}^{-\mathbf{1}} \mathbf{h} .
$$

Setting the gradient vector of $f_{\mathrm{MAP}}^{(m)}(\mathbf{h}, \epsilon)$ with respect to $\mathbf{h}^{H}$ to zero produces the channel estimate

$$
\widehat{\mathbf{h}}_{\Delta \theta_{m}}=\left[\mathbf{S}_{\Delta \theta_{m}}^{H} \mathbf{S}_{\Delta \theta_{m}}+\sigma_{g}^{2} \mathbf{R}_{\mathbf{h}}^{-1}\right]^{-1} \mathbf{S}_{\Delta \theta_{m}}^{H} \boldsymbol{\Phi}_{\Delta \theta_{m}, \epsilon}^{H} \mathbf{r}_{\Delta \theta_{m}} .
$$

Replacing Equation 32 into Equation 31 yields the CFO estimate

$$
\hat{\epsilon}_{\Delta \theta_{m}}=\arg \min _{\epsilon} g_{\mathrm{MAP}}^{(m)}(\epsilon),
$$

where

$$
g_{\mathrm{MAP}}^{(m)}(\epsilon)=\mathbf{r}_{\Delta \theta_{m}}^{H} \boldsymbol{\Phi}_{\Delta \theta_{m}, \epsilon} \mathbf{S}_{\Delta \theta_{m}}^{+} \boldsymbol{\Phi}_{\Delta \theta_{m}, \epsilon}^{H} \mathbf{r}_{\Delta \theta_{m}},
$$

with

$$
\mathbf{S}_{\Delta \theta_{m}}^{+}=\mathbf{S}_{\Delta \theta_{m}}\left[\mathbf{S}_{\Delta \theta_{m}}^{H} \mathbf{S}_{\Delta \theta_{m}}+\mathbf{R}_{\mathbf{h}}^{-\mathbf{1}} \sigma_{g}^{2}\right]^{-1} \mathbf{S}_{\Delta \theta_{m}}^{H} .
$$

To estimate $\hat{\epsilon}_{\Delta \theta_{m}}$, an iterative algorithm based on the Newton-Raphson method is designed as follows:

$$
\hat{\epsilon}_{\Delta \theta_{m}, i+1}=\hat{\epsilon}_{\Delta \theta_{m}, i}-\left.\left[\frac{\partial^{2} g_{\mathrm{MAP}}^{(m)}(\epsilon)}{\partial \epsilon^{2}}\right]^{-1} \frac{\partial g_{\mathrm{MAP}}^{(m)}(\epsilon)}{\partial \epsilon}\right|_{\epsilon=\hat{\epsilon}_{\Delta \theta_{m}, i}},
$$

where $\hat{\epsilon}_{\Delta \theta_{m}, i}$ indicates the CFO estimate at the $i$ th iteration, and

$$
\begin{aligned}
\frac{\partial g_{\mathrm{MAP}}^{(m)}(\epsilon)}{\partial \epsilon}= & 2 \Re\left\{\mathbf{r}_{\Delta \theta_{m}}^{H} \mathbf{Q}_{\Delta \theta_{m}} \boldsymbol{\Phi}_{\Delta \theta_{m}, \epsilon} \mathbf{S}_{\Delta \theta_{m}}^{+} \boldsymbol{\Phi}_{\Delta \theta_{m}, \epsilon}^{H} \mathbf{r}_{\Delta \theta_{m}}\right\}, \\
\frac{\partial g_{\mathrm{MAP}}^{2}(\epsilon)}{\partial \epsilon^{2}}= & 2 \Re\left\{\mathbf{r}_{\Delta \theta_{m}}^{H} \mathbf{Q}_{\Delta \theta_{m}}^{2} \boldsymbol{\Phi}_{\Delta \theta_{m}, \epsilon} \mathbf{S}_{\Delta \theta_{m}}^{+} \boldsymbol{\Phi}_{\Delta \theta_{m}, \epsilon}^{H} \mathbf{r}_{\Delta \theta_{m}}+\right. \\
& \left.+\mathbf{r}_{\Delta \theta_{m}}^{H} \mathbf{Q}_{\Delta \theta_{m}} \boldsymbol{\Phi}_{\Delta \theta_{m}, \epsilon} \mathbf{S}_{\Delta \theta_{m}}^{+} \mathbf{Q}_{\Delta \theta_{m}}^{H} \boldsymbol{\Phi}_{\Delta \theta_{m}, \epsilon}^{H} \mathbf{r}_{\Delta \theta_{m}}\right\}, \\
\mathbf{Q}_{\Delta \theta_{m}}= & j \frac{2 \pi}{N} \operatorname{diag}\left\{n-\Delta \theta_{m}, \ldots, n-\Delta \theta_{m}+2 N+N_{S}\right. \\
& \left.+N_{G}-1\right\} .
\end{aligned}
$$

The iterative process in Equation 34 requires an initialization with some frequency offset value $\hat{\epsilon}_{\Delta \theta_{m}, 0}$. If the initial value (taken randomly) is far from the true frequency offset value, the synchronization algorithm performance can be strongly affected since the function $g_{\mathrm{MAP}}^{(m)}(\epsilon)$ may have several local minima. To avoid this situation, we propose to initialize the Newton-Raphson iteration with a coarse value close to the true frequency offset provided by the ACF as mentioned in $[18,19]$. Based on the STF, the coarse CFO estimation $\epsilon_{c}$ is performed according to Equation 6 while the fine CFO estimation $\epsilon_{f}$ is given by Equation 7 relying on the LTF. The frequency offset estimate, given by Equation $8, \hat{\epsilon}=\epsilon_{c}+\epsilon_{f}$, is then considered as the starting frequency offset value $\hat{\epsilon}_{\Delta \theta_{m}, 0}$. Although our algorithm adds one step before the Newton-Raphson iterations to get a more appropriate initial value $\epsilon_{0}$, this process is efficient since the number of iterations to obtain the final estimate is reduced. 
Substituting $\hat{\epsilon}_{\Delta \theta_{m}}$ into Equation 32 gives the CIR estimate

$$
\widehat{\mathbf{h}}_{\Delta \theta_{m}}=\left[\mathbf{S}_{\Delta \theta_{m}}^{H} \mathbf{S}_{\Delta \theta_{m}}+\sigma_{g}^{2} \mathbf{R}_{\mathbf{h}}^{-\mathbf{1}}\right]^{-1} \mathbf{S}_{\Delta \theta_{m}}^{H} \boldsymbol{\Phi}_{\Delta \theta_{m}, \hat{\epsilon}_{m}}^{H} \mathbf{r}_{\Delta \theta_{m}}
$$

Note that $h_{\max }^{0}=\max _{\Delta \theta_{i}}\left|\hat{h}_{\Delta \theta_{i}}(0)\right|,-M \leq i \leq M$. Among the $2 M+1$ estimates of $\widehat{\mathbf{h}}_{\Delta \theta_{m}}$, we select the one that satisfies the following conditions:

$$
\left|\hat{h}_{\Delta \theta_{m}}(0)\right|>\beta h_{\max }^{0}
$$

where $\beta$ is a given threshold, smaller than 1 , depending on the channel model $\frac{|h(L-1)|}{|h(0)|}$. The set $\boldsymbol{\Lambda}$ now becomes $\boldsymbol{\Gamma}=\left\{\omega_{0}, \ldots, \omega_{M^{\prime}} ; M^{\prime} \leq 2 M\right\}$ and finally the correct time offset is estimated by

$$
\Delta \widehat{\theta}=\arg \max _{\omega_{m^{\prime}}} \sum_{n=0}^{L-1}\left|\hat{h}_{\omega_{m^{\prime}}}(n)\right|^{2}
$$

\subsection{Third stage: frequency domain-based fine timing estimation}

The second stage provides an estimate of CFO which is used to compensate the frequency offset of the received signal $r(n)$ corresponding to LTF sequence as follows:

$$
\begin{aligned}
r_{e}(n) & =r(n) \times e^{-j \frac{2 \pi \widehat{\epsilon}}{N}} \\
& =\sum_{i=0}^{L-1} h(i) x\left(n-i-\Delta \theta_{e}\right) e^{j \frac{2 \pi \epsilon \Delta \theta_{e}}{N}} \times e^{\left.-j \frac{2 \pi(\widehat{\epsilon}-\epsilon)}{N}\right)}+g^{\prime}(n),
\end{aligned}
$$

where $\Delta \theta_{e}=\Delta \widehat{\theta}-\Delta \theta$ is a possible remaining time offset (Section 4.3). According to the results provided in [24], the following approximation is performed $e^{\left.-j \frac{2 \pi(\hat{\epsilon}-\epsilon)}{N}\right)} \approx$ 1. Therefore, the frequency offset compensated signal becomes:

$$
r_{e}(n) \approx \sum_{i=0}^{L-1} h(i) x\left(n-i-\Delta \theta_{e}\right) e^{j \frac{2 \pi \epsilon \Delta \theta_{e}}{N}}+g^{\prime}(n) .
$$

For convenience, the received signal $r_{e}(n) \approx$ $\sum_{i=0}^{L-1} h_{\Delta \theta_{e}, \epsilon}(i) x\left(n-i-\Delta \theta_{e}\right)+g^{\prime}(n)$ is expressed in frequency domain in a matrix form as follows:

$$
\mathbf{R}_{e}=\mathbf{X} \mathbf{H}_{\Delta \boldsymbol{\theta}_{e}, \epsilon}+\mathbf{G},
$$

where $\mathbf{X}$ contains the known LTF of the physical packet in the frequency domain, and $\mathbf{G}$ is the noise vector of length $N$. To simplify notations for the rest of the paper, $\mathbf{H}_{\Delta \boldsymbol{\theta}_{e}, \epsilon}$ is replaced by $\mathbf{H}$ being the true CIR in the frequency domain and is assumed to follow a circular Gaussian distribution with zero mean, given by
$\Psi(\mathbf{H})=\frac{1}{\pi^{N} \operatorname{det}\left(\mathbf{R}_{\mathbf{H}}\right)} \exp \left(\mathbf{H}^{H} \mathbf{R}_{\mathbf{H}}{ }^{-1} \mathbf{H}_{\boldsymbol{\Delta} \boldsymbol{\theta}_{e}, \epsilon}\right)$ where $\mathbf{R}_{\mathbf{H}}$ is the covariance matrix of size $N \times N$.

The performance analysis in terms of synchronization failure probability provided in $[20,22]$ shows that the accuracy of the time offset estimation depends heavily on the channel estimation $\hat{\mathbf{H}}$. However, since one knows the method which has been used for channel estimation, one has the knowledge of the estimate given the true channel. Therefore, it becomes feasible to compute the time offset as an expectation of all possible true channels given the estimated one, which corresponds to a minimization of the following new metric:

$$
\Delta \hat{\theta}_{e}=\arg \min _{\Delta \theta_{e} \in \Lambda}\left\{\widetilde{D}\left(\Delta \theta_{e}\right)\right\}
$$

where $\widetilde{D}\left(\Delta \theta_{e}\right)$ is the average of the transmission error function (TEF), $D(\mathbf{H})=\left\|\mathbf{R}_{e}-\mathbf{X} \mathbf{H}\right\|^{2}$, over all channel estimation errors, as given by

$$
\widetilde{D}\left(\Delta \theta_{e}\right)=E_{\mathbf{H} \mid \hat{\mathbf{H}}}[D(\mathbf{H})]=\int_{\mathbf{H}} D(\mathbf{H}) \Psi(\mathbf{H} \mid \hat{\mathbf{H}}) d(\mathbf{H}),
$$

where $\Psi(\mathbf{H} \mid \hat{\mathbf{H}})$ is the pdf of $\mathbf{H}$ given $\hat{\mathbf{H}}$, and $\hat{\mathbf{H}}$ is estimated by $\hat{\mathbf{H}}=\mathbf{X}^{-1} \mathbf{R}_{e}$. Note that the form of this equation has been inspired from [29].

To solve Equation 42, the knowledge of $\Psi(\mathbf{H} \mid \hat{\mathbf{H}})$ is required and is simply obtained by applying Bayes's formula $\Psi(\mathbf{H} \mid \hat{\mathbf{H}})=\Psi(\hat{\mathbf{H}} \mid \mathbf{H}) \Psi(\mathbf{H}) / \Psi(\hat{\mathbf{H}})$, where $\Psi(\hat{\mathbf{H}} \mid \mathbf{H})=$ $C \aleph\left(\boldsymbol{\mu}_{\hat{\mathbf{H}} \mid \mathbf{H}}, \boldsymbol{\Sigma}_{\hat{\mathbf{H}} \mid \mathbf{H}}\right)$ and $\Psi(\hat{\mathbf{H}})=C \aleph\left(\boldsymbol{\mu}_{\hat{\mathbf{H}}}, \boldsymbol{\Sigma}_{\hat{\mathbf{H}}}\right)$ and $\Psi(\mathbf{H} \mid \hat{\mathbf{H}})=$ $C \aleph\left(\boldsymbol{\mu}_{\mathbf{H} \mid \hat{\mathbf{H}}}, \boldsymbol{\Sigma}_{\mathbf{H} \mid \hat{\mathbf{H}}}\right)$. The mean and the variance of $\Psi(\hat{\mathbf{H}} \mid \mathbf{H})$ can be rewritten as follows: $\boldsymbol{\mu}_{\hat{\mathbf{H}} \mid \mathbf{H}}=E\left\{\left.\hat{\mathbf{H}}\right|_{\mathbf{H}}\right\}=E\{(\mathbf{H}+$ $\left.\left.\mathbf{X}^{-1} \mathbf{G}\right)\left.\right|_{\mathbf{H}}\right\}=E\left\{\left.\mathbf{H}\right|_{\mathbf{H}}\right\}=\boldsymbol{\mu}_{H}=0$, and $\boldsymbol{\Sigma}_{\hat{\mathbf{H}} \mid \mathbf{H}}=E\left\{\left.\hat{\mathbf{H}} \hat{\mathbf{H}}^{H}\right|_{\mathbf{H}}\right\}=$ $E\left\{\left.\left(\mathbf{H}+\mathbf{X}^{-\mathbf{1}} \mathbf{G}\right)\left(\mathbf{H}+\mathbf{X}^{-\mathbf{1}} \mathbf{G}\right)^{H}\right|_{\mathbf{H}}\right\}-\boldsymbol{\mu}_{\hat{\mathbf{H}} \mid \mathbf{H}} \boldsymbol{\mu}_{\hat{\mathbf{H}} \mid \mathbf{H}}^{H}=$ $E\left(\left(\mathbf{X}^{-\mathbf{1}} \mathbf{G}\right)^{H}\left(\mathbf{X}^{-\mathbf{1}} \mathbf{G}\right)\right) \mathbf{I}=\mathbf{\Sigma}_{\epsilon}$, where $\mathbf{I}$ is $N \times N$ identity matrix. Then, $\Psi(\hat{\mathbf{H}})=C \aleph\left(0, \mathbf{R}_{\mathbf{H}}+\boldsymbol{\Sigma}_{\epsilon}\right)$ where $\mathbf{R}_{\mathbf{H}}=E\left\{\mathbf{H} \mathbf{H}^{H}\right\}$ is approximated by $\mathbf{R}_{\mathbf{H}} \approx E\left\{\hat{\mathbf{H}}_{\mathrm{MAP}} \hat{\mathbf{H}}_{\mathrm{MAP}}^{H}\right\}$ ( $\hat{\mathbf{H}}_{\text {MAP }}$ given by Equation 24). Hence, $\boldsymbol{\mu}_{\mathbf{H} \mid \hat{\mathbf{H}}}=\boldsymbol{\Sigma}_{\Delta} \hat{\mathbf{H}}$ and $\boldsymbol{\Sigma}_{\mathbf{H} \mid \hat{\mathbf{H}}}=\boldsymbol{\Sigma}_{\Delta} \Sigma_{\epsilon}$ where $\boldsymbol{\Sigma}_{\Delta}=\mathbf{R}_{\mathbf{H}}\left(\mathbf{R}_{\mathbf{H}}+\boldsymbol{\Sigma}_{\epsilon}\right)^{-1}$. Therefore, Equation 42 is reduced to

$$
\widetilde{D}\left(\Delta \theta_{e}\right)=E_{\mathbf{W}}[D(\mathbf{W})]=\int_{\mathbf{W}} D(\mathbf{W}) \Psi(\mathbf{W}) d(\mathbf{W}),
$$

where $\Psi(\mathbf{W})=C \aleph\left(\boldsymbol{\mu}_{\mathbf{H} \mid \hat{\mathbf{H}}}, \boldsymbol{\Sigma}_{\mathbf{H} \mid \hat{\mathbf{H}}}\right)$ and $D(\mathbf{W})=\| \mathbf{R}_{e}-$ $\mathbf{X W} \|^{2}$. After some mathematical manipulations, we obtain

$$
\begin{aligned}
\widetilde{D}\left(\Delta \theta_{e}\right)= & E\left[\mathbf{R}_{e}^{H} \mathbf{R}_{e}\right]-E\left[\mathbf{R}_{e}^{H} \mathbf{X}\right] \boldsymbol{\mu}_{\mathbf{H} \mid \hat{\mathbf{H}}}-\boldsymbol{\mu}_{\mathbf{H} \mid \hat{\mathbf{H}}}^{H} E\left[\mathbf{X}^{H} \mathbf{R}_{e}\right] \\
& +\operatorname{trace}\left(\boldsymbol{\Sigma}_{\mathbf{H} \mid \hat{\mathbf{H}}} \mathbf{X} \mathbf{X}^{H}\right)+\boldsymbol{\mu}_{\mathbf{H} \mid \hat{\mathbf{H}}}^{H} \mathbf{X}^{H} \mathbf{X} \boldsymbol{\mu}_{\mathbf{H} \mid \hat{\mathbf{H}}}
\end{aligned}
$$


where trace $(\cdot)$ indicates the trace operator. The remaining time offset estimate $\Delta \hat{\theta}_{e}$ is then deduced from Equation 41.

\section{Performance evaluation and discussions}

This section discusses the performance of the proposed DA-NDA time and frequency multistage synchronization algorithm in terms of probability of synchronization failure (PSF) and bit error ratio (BER). Table 1 lists the simulation parameters compliant with the IEEE 802.11a standard [1]. The tolerance of the internal oscillator at each station belongs to the range $[-20,20] \mathrm{ppm}$, and thus the total tolerance of two stations falls in $[-40,40] \mathrm{ppm}$. For a carrier frequency of $f_{c}=5.2 \mathrm{GHz}$ and an OFDM symbol of duration $T=N \times T_{s}=3.2 \mu \mathrm{s}$, the normalized CFO $(\epsilon)$ falls in the range $[-0.6,0.6] . \epsilon$ and $\theta$ are taken randomly according to a uniform distribution. Two channel models are tested: COST-207 RA [30] and BRAN-A (CH-A) [31]. The threshold $\beta$ is set to 0.35 (average value).

The performance of the proposed algorithm is compared to that of the algorithm described in Section 3 [19]. This choice is based on the fact that this algorithm is dedicated to the 802.11a standard and was shown better performance than other algorithms developed in the literature. Moreover, it is implemented by the initialization stage of our algorithm. In order to demonstrate the efficiency of our algorithm, we also consider several possible configurations depending on whether both offsets (time and frequency) are estimated, or if only one but the other is perfectly known by the receiver. Moreover, we discuss the contribution of stages 2 and 3 to the performance of our synchronization algorithm. We also analyze the initialization stage impact: (a) no RtS/CtS synchronization; and (b) RtS/CtS synchronization included with a power level deployed to transmit RtS/CtS control frames equal or twice that of the nominal transmission power level used to transmit DATA frames.

Table 1 Simulation parameters

\begin{tabular}{ll}
\hline Parameters & Values \\
\hline Bandwidth $(B)$ & $20 \mathrm{MHz}$ \\
Sampling time $\left(T_{s}\right)$ & $50 \mathrm{~ns}$ \\
Number of subcarriers $\left(N_{C}\right)$ & 52 \\
Number of points FFT/IFFT $(N)$ & 64 \\
Subcarrier spacing $(\Delta F)$ & $0.3125 \mathrm{MHz}$ \\
Data rate & $6 \mathrm{Mbps}$ \\
LSTF & 160 \\
LSIG & 80 \\
$K$ & 80 \\
$M$ & 30 \\
\hline
\end{tabular}

The performance of the synchronization algorithms listed below is compared:

i) Algorithm '[19]' (described in Section 3) where ACF is applied on STF for CTS and FS, followed by CCF using LTF for FTS stage.

ii) Algorithm '[19] with a perfect TS' concerns the algorithm in (i) where the symbol timing true value $\theta$ is assumed to be known by the receiver and is then used to compensate the time offset perfectly.

iii) Algorithm '[19] with a perfect FS' concerns the algorithm in (i) where the frequency offset true value $\epsilon$ is assumed to be known by the receiver and is then used to compensate the frequency offset perfectly.

iv) 'Joint MAP TS-FS' is the algorithm developed in [24]. It concerns the first two stages ('Stage 1' and 'Stage 2' provided in Figure 3): (a) CTS stage presented in Section 4.2 exploiting not only the channel information but also the SIGNAL field; and (b) joint MAP frequency and time synchronization as described in Section 4.3.

v) 'Proposed algo., no synch. in init. stage' is the proposed synchronization algorithm where the stations are assumed to be synchronized during the negotiation of the transmission medium reservation. Meaning that the synchronization problem is not considered in the initialization stage.

vi) 'Proposed algo.' is the proposed synchronization algorithm performed in a realistic transmission scenario (initialization stage, followed by three stages) where the synchronization of stations during the negotiation of the transmission medium reservation is considered. Two situations are analyzed when the power level used to transmit RTS/CTS control frames is: (a) the same as the nominal transmission power level of the DATA frame (i.e. difference of $0 \mathrm{~dB}$ ); and (b) twice than the nominal transmission power level of the DATA frame (i.e. difference of $3 \mathrm{~dB}$ ).

vii) 'Joint MAP TS-FS with a perfect TS' is the algorithm in (iv) where the symbol timing true value $\theta$ is assumed to be known by the receiver and is then used to compensate the time offset perfectly.

viii) 'Joint MAP TS-FS with a perfect FS' is the algorithm in (iv) where the frequency offset true value $\epsilon$ is assumed to be known and is then used to compensate the frequency offset perfectly. Specifically, the received signal after CTS stage (i.e., 'Stage 1' in Figure 3) given by Equation 28 is multiplied by $e^{-j 2 \pi \epsilon n / N}$. Therefore, Equation 29 is reduced to $\mathbf{r}=\mathbf{S}_{\Delta \theta} \mathbf{h}+\mathbf{g}$ since $\boldsymbol{\Phi}_{\Delta \theta, \epsilon}$ becomes an identity matrix. The problem to be considered returns then to a joint MAP time and channel estimation where Equation 30 becomes $\{\hat{\mathbf{h}}, \Delta \widehat{\theta}\}=\arg \max _{\mathbf{h}, \Delta \theta} \ln P(\mathbf{h}, \Delta \theta \mid \mathbf{r})$. To solve this equation, a set $\boldsymbol{\Lambda}$ containing $2 M+1$ 
possible time offset values (i.e., $\boldsymbol{\Lambda}=\left\{-\Delta \theta_{M}, \ldots\right.$, $\left.\left.\Delta \theta_{M}\right\}\right)$ is defined. For a given value $\Delta \theta_{m} \in \boldsymbol{\Lambda}$, the MAP-based estimation of channel coefficients is given by $\left\{\hat{\mathbf{h}}_{\Delta \theta_{m}}\right\}=\arg \min _{\mathbf{h}} f_{\mathrm{MAP}}^{(m)}(\mathbf{h})$, with $f_{\mathrm{MAP}}^{(m)}(\mathbf{h})=$ $\frac{1}{\sigma_{g}^{2}}\left\|\mathbf{r}_{\Delta \theta_{m}}-\mathbf{S}_{\Delta \theta_{m}} \mathbf{h}\right\|^{2}+\mathbf{h}^{H} \mathbf{R}_{\mathbf{h}}^{-1} \mathbf{h}$. Setting the gradient vector of $f_{\text {MAP }}^{(m)}(\mathbf{h})$ with respect to $\mathbf{h}^{H}$ to zero provides the MAP-based channel estimate $\widehat{\mathbf{h}}_{\Delta \theta_{m}}=\left[\mathbf{S}_{\Delta \theta_{m}}^{H}\right.$ $\left.\mathbf{S}_{\Delta \theta_{m}}+\sigma_{g}^{2} \mathbf{R}_{\mathbf{h}}^{-\mathbf{1}}\right]^{-1} \mathbf{S}_{\Delta \theta_{m}}^{H} \mathbf{r}_{\Delta \theta_{m}}$. Equations 36 and 37 are then performed to obtain $\Delta \widehat{\theta}$.

Simulation results are provided in Figures 5, 6, 7, 8, 9, 10, 11 , and 12 . Figures $5,7,9$, and 11 deal with the COST207RA channel model while the other ones concern with the BRAN-A channel model. When available, a box in the plot indicates the SNR region of interest in the actual standard. This will demonstrate that similar performance can be obtained with our algorithm for much smaller SNRs.

Note that the Newton-Raphson method, in the second stage, requires no more than five iterations in the worst case (i.e., for very low SNR). Indeed, its initial value is chosen as a coarse value close to the true frequency offset value provided by the ACF, as in $[18,19]$.

Figures 5 and 6 provide the MSE calculated between the true CFO and its estimate $\left(E\left\{\left(\epsilon-\hat{\epsilon}_{m}\right)^{2}\right\}\right)$ versus SNR. The results show that the MSE provided by our algorithm is much smaller than those provided by other algorithms even in the case where our algorithm addresses the synchronization problem of the control frames and that these frames are sent with identical power level than DATA frames. Consider the operating mode of the IEEE 802.11a, for instance in Figure 5 at SNR $=17.5 \mathrm{~dB}$, we obtain a

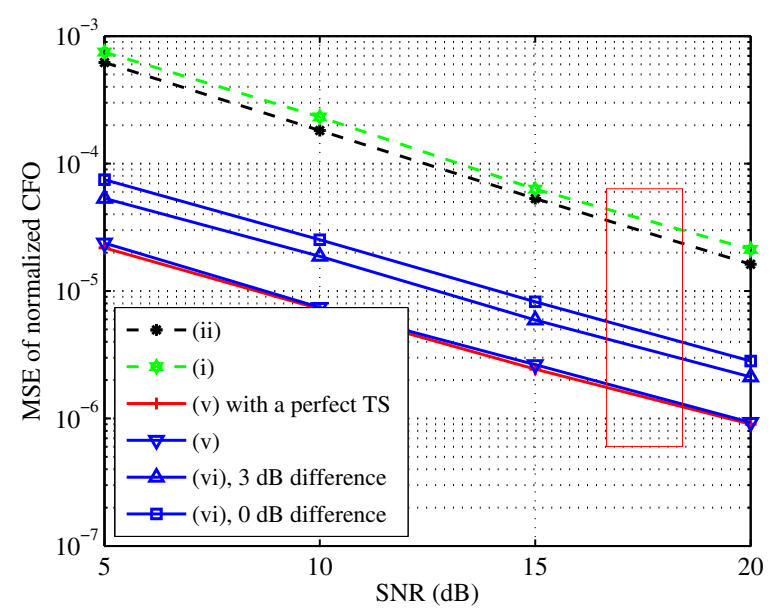

Figure 5 MSE of normalized CFO under COST207-RA channel model. The rectangular box represents the operating area of the 802.11a standard.

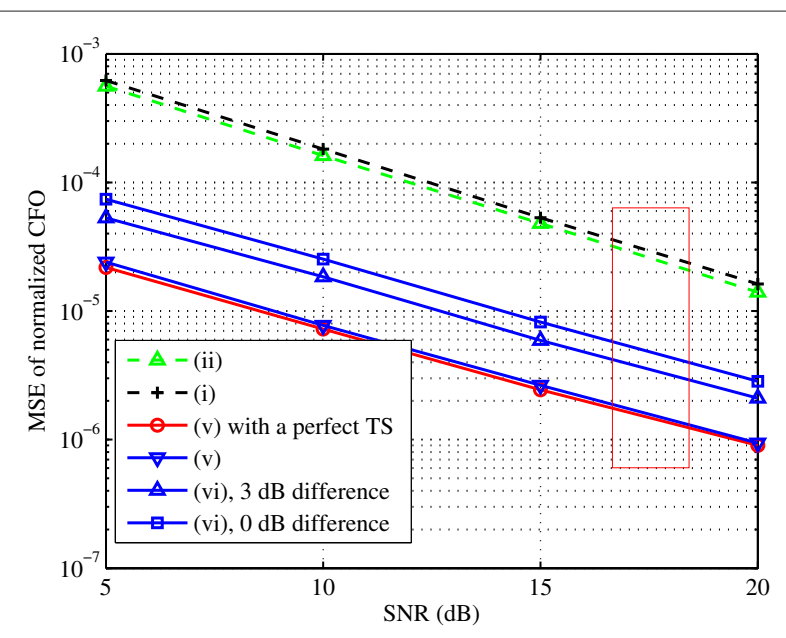

Figure 6 MSE of normalized CFO under BRAN-A channel.

MSE of $3.8 \times 10^{-5}$ for the algorithm '[19]', of $1.6 \times 10^{-6}$ for 'Proposed algo. no synch. in init. stage', of $3.5 \times 10^{-6}$ for 'Proposed algo., $0 \mathrm{~dB}$ difference', and of $5 \times 10^{-6}$ for 'Proposed algo., $3 \mathrm{~dB}$ difference.' Moreover regardless of the time synchronization being perfect or not, the two curves associated to our algorithm (with no synch. in init. stage) are similar, under both the COST207-RA and the BRAN-A channel models.

Figures 7 and 8 measure the detection probability of arrival time of the transmitted physical packet for a given deviation with respect to its true time position (i.e., $\widehat{\theta}-\theta$ ) at SNR $=15 \mathrm{~dB}$ for '[19]', 'Joint MAP TS-FS', and 'Proposed algo. no synch. in init. stage' algorithms using $7 \times 10^{4}$ realizations under COST207RA and $10^{7}$ realizations under BRAN-A. The 'Proposed algo., $3 \mathrm{~dB}$ difference' achieves the highest estimation accuracy on the two channel models when the packet arrival time is detected without time deviation (with respect to the arrival time of packet) compared to other algorithms.

Figures 9 and 10 refer to the PSF versus SNR. The comparison results show that our algorithms (i.e. 'Proposed algo. $3 \mathrm{~dB}$ difference' and 'Proposed algo. $0 \mathrm{~dB}$ difference') give better performance regardless the selected channel model. Note that it is also possible to accept packets whose arrival time is estimated after the true position with a deviation (different from zero) due to the use of CP [32]. When using the COST207-RA channel model, we can accept arrival packet with a delay no more than four samples (i.e., $\widehat{\theta}_{\text {new }}=\widehat{\theta}-4$ ). This timing delay is accepted since the CP of the OFDM symbol and the maximum delay of the channel response are respectively equal to 16 and 13 samples, and moreover the average power of tap 0 is a hundred times that of tap 12 (i.e., $P_{\text {ave }}(0)=100 \times$ $\left.P_{\text {ave }}(12)\right)$. For the same reasons, when using the BRAN-A 


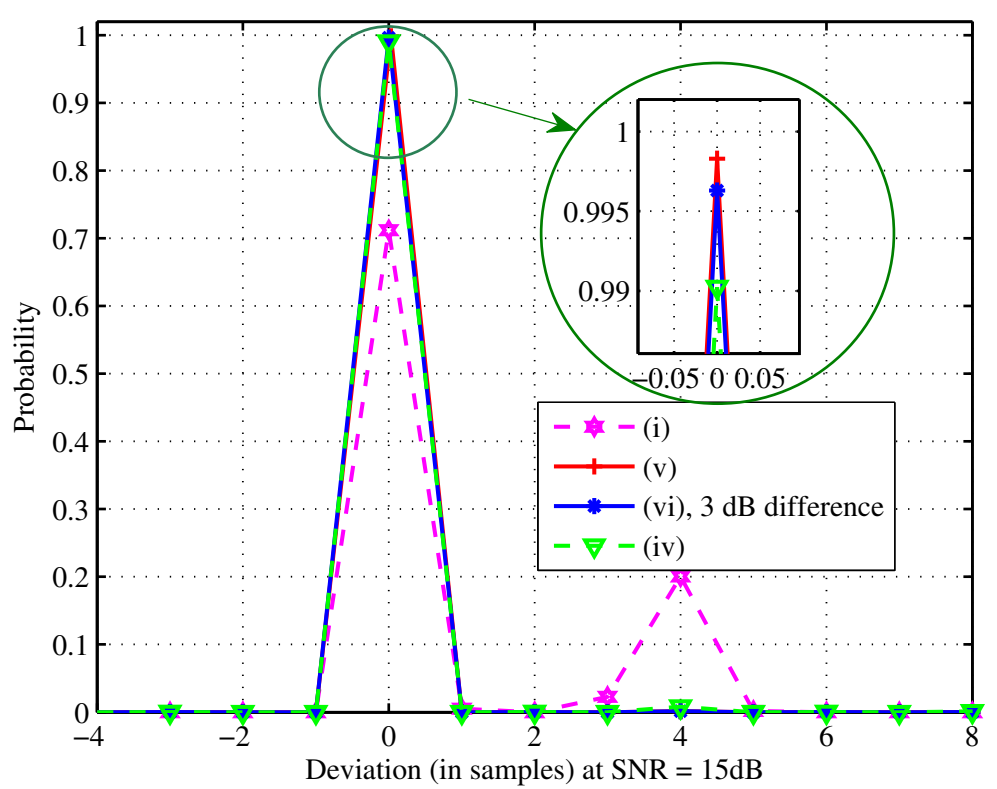

Figure 7 Deviation with respect to the true time position of a physical packet under COST207-RA channel.

model, it is possible to accept an arrival packet with a delay no more than seven samples (i.e., $\widehat{\theta}_{\text {new }}=\widehat{\theta}-7$ ). In both cases, the orthogonality of subcarrier frequency components is completely preserved in spite of the fact that there exists a phase offset which is however compensated by a single-tap frequency-domain equalizer [33]. With a time deviation of no more than the maximum number of samples tolerated by each channel model, the PSF of both algorithms is reduced. However, the PSF of our algorithm is smaller than that of the other algorithms. For example in Figure 9 at $\mathrm{SNR}=17.5 \mathrm{~dB}$ and with time deviation no more than 4 , we read a PSF of $7 \times 10^{-3}$ for '[19]', of $10^{-3}$ for 'Joint MAP TS-FS', of $6 \times 10^{-5}$ for 'Proposed algo., no synch. in init.stage', of $2 \times 10^{-4}$ for 'Proposed algo., 3 $\mathrm{dB}$ difference,' and of $3.3 \times 10^{-4}$ for 'Proposed algo., $0 \mathrm{~dB}$ difference.'

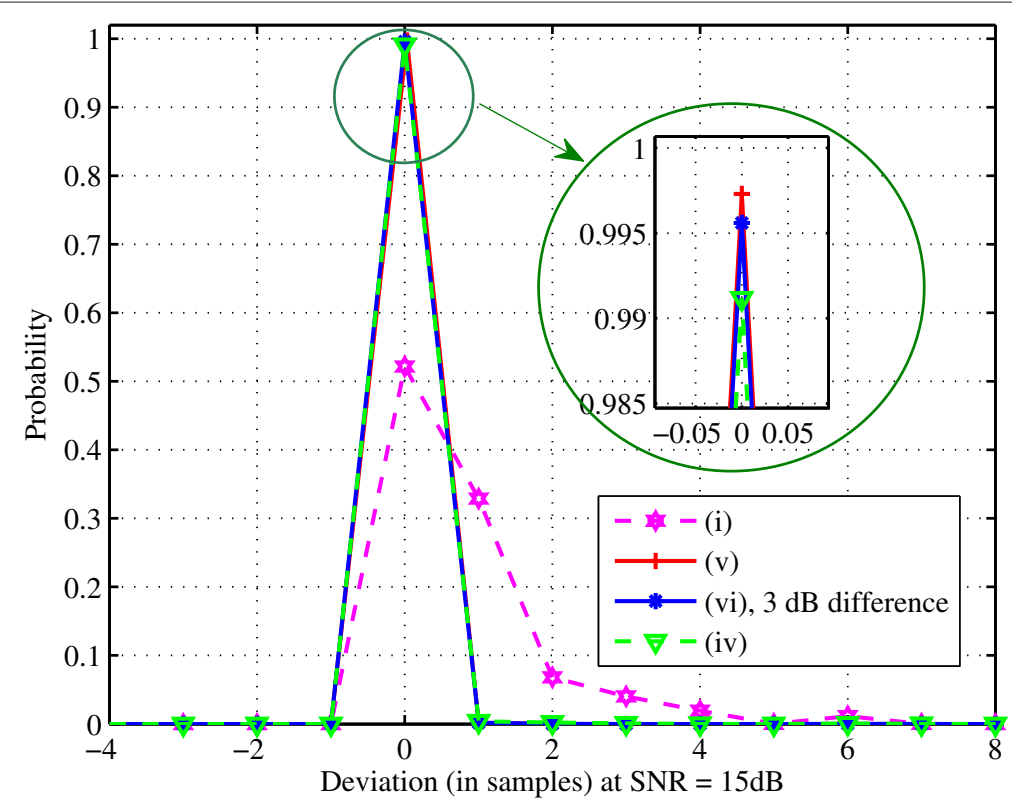

Figure 8 Deviation with respect to the true time position of a physical packet under BRAN-A channel. 


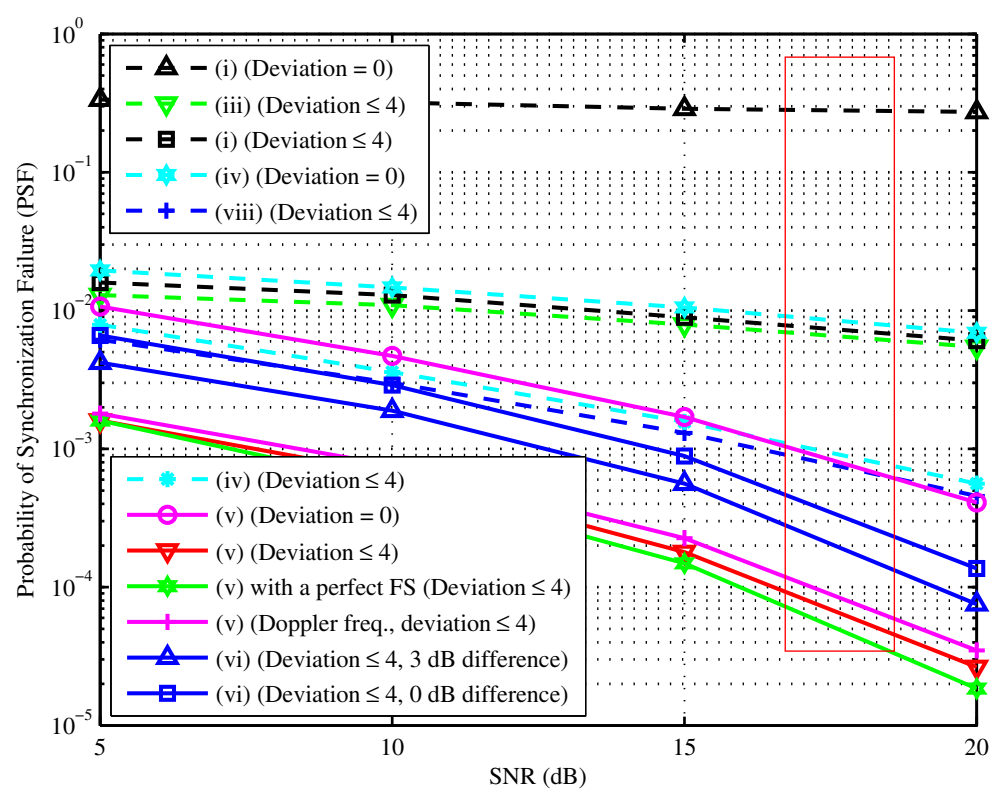

Figure 9 PSF under COST207-RA channel.

In Figure 10 at $\mathrm{SNR}=17.5 \mathrm{~dB}$ with a time deviation of no more than 7 , when the CFO is not perfectly compensated, we obtain a PSF of only $3 \times 10^{-6}$ for 'Proposed algo., no synch. in init. stage,' $2.3 \times 10^{-5}$ for 'Proposed algo., 0 $\mathrm{dB}$ difference, and $1.2 \times 10^{-5}$ for 'Proposed algo., $3 \mathrm{~dB}$ difference' much lower than that for '[19]' $\left(7 \times 10^{-3}\right)$ and for 'Joint MAP TS-FS' $\left(3 \times 10^{-4}\right)$. Moreover, the PSF results indicate that the performance of our algorithm in both cases (i.e., perfect or imperfect FS) is almost the same.
Indeed, this is proved by the deployment of some mathematical equations as follows. Substituting Equation 29 (i.e., $\mathbf{r}_{\Delta \theta_{m}}=\boldsymbol{\Phi}_{\Delta \theta_{m}, \epsilon} \mathbf{S}_{\Delta \theta_{m}} \mathbf{h}+\mathbf{g}$ ) into Equation 35 leads to

$$
\begin{aligned}
\widehat{\mathbf{h}}_{\Delta \theta_{m}}= & {\left[\mathbf{S}_{\Delta \theta_{m}}^{H} \mathbf{S}_{\Delta \theta_{m}}+\sigma_{g}^{2} \mathbf{R}_{h}^{-1}\right]^{-1} \mathbf{S}_{\Delta \theta_{m}}^{H} \mathbf{I}_{\Delta \theta, \hat{\epsilon}_{m}}\left(\mathbf{S}_{\Delta \theta_{m}} \mathbf{h}\right) } \\
& +\left[\mathbf{S}_{\Delta \theta_{m}}^{H} \mathbf{S}_{\Delta \theta_{m}}+\sigma_{g}^{2} \mathbf{R}_{h}^{-1}\right]^{-1} \mathbf{S}_{\Delta \theta_{m}}^{H} \mathbf{\Phi}_{\Delta \theta_{m}, \hat{\epsilon}_{m}} \mathbf{g}
\end{aligned}
$$

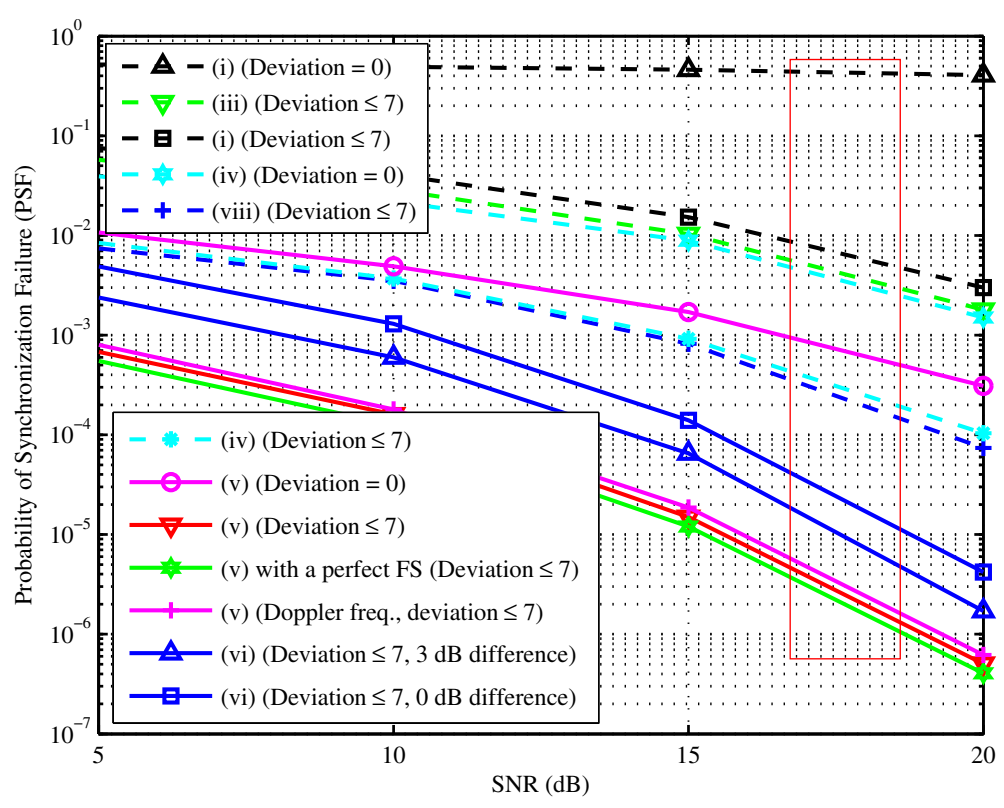

Figure 10 PSF under BRAN-A channel. 


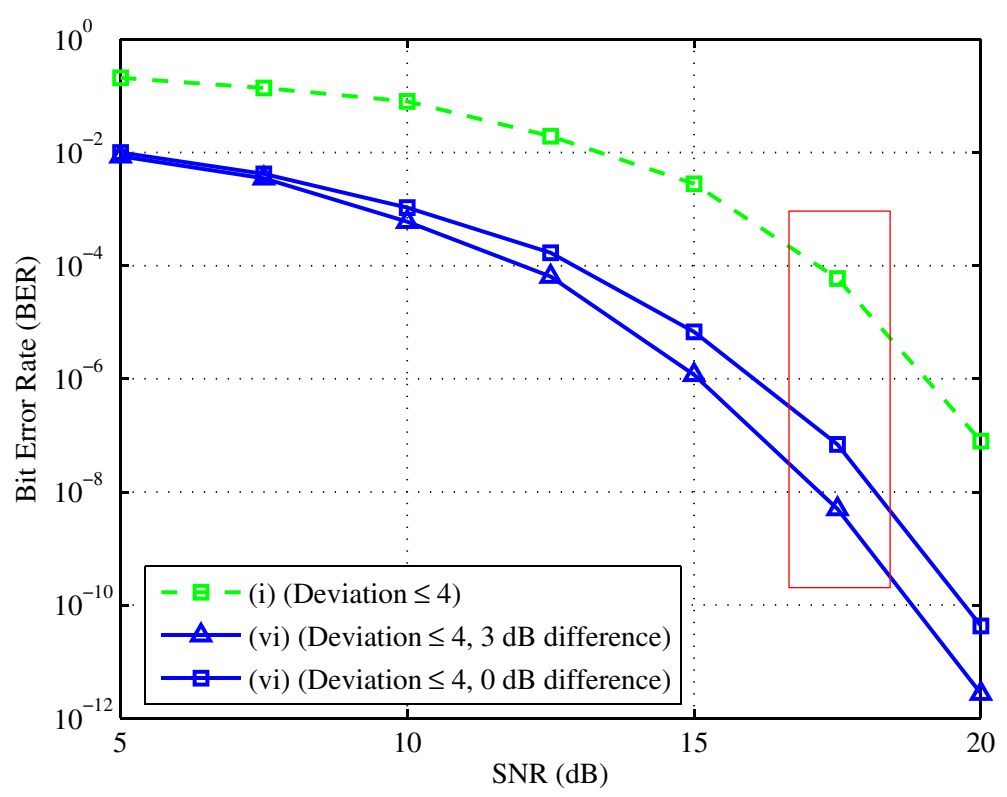

Figure 11 BER under COST207-RA channel.

where $\mathbf{I}_{\Delta \theta, \hat{\epsilon}_{m}}$ is the diagonal matrix of size $(2 N+$ $\left.N_{G}+N_{S}\right) \times\left(2 N+N_{G}+N_{S}\right)$ and is given by $\mathbf{I}_{\Delta \theta, \hat{\epsilon}_{m}}=$ $\operatorname{diag}\left\{e^{j 2 \pi\left(\epsilon-\hat{\epsilon}_{m}\right) \Delta \theta / N}, e^{j 2 \pi\left(\epsilon-\hat{\epsilon}_{m}\right) \Delta \theta / N}, \ldots, e^{j 2 \pi\left(\epsilon-\hat{\epsilon}_{m}\right) \Delta \theta / N}\right\}$. Moreover, Figures 5 and 6 show that for a given $\mathrm{SNR}=17.5 \mathrm{~dB}$, the MSE between the normalized CFO and its true value (i.e., $\left.E\left\{\left(\epsilon-\hat{\epsilon}_{m}\right)^{2}\right\}\right)$ generated by our algorithm is equal to $2.7 \times 10^{-6}$ under COST207-RA and $1.6 \times 10^{-6}$ under BRAN-A. The experimental results also show that the remaining time offset value $\Delta \theta$ is relatively small, leading to the following approximation $e^{j 2 \pi\left(\epsilon-\hat{\epsilon}_{m}\right) \Delta \theta / N} \approx 1$. Consequently, $\mathbf{I}_{\Delta \theta, \hat{\epsilon}_{m}}$ is considered as an identity matrix and Equation 45 becomes

$$
\widehat{\mathbf{h}}_{\Delta \theta_{m}}=\left[\mathbf{S}_{\Delta \theta_{m}}^{H} \mathbf{S}_{\Delta \theta_{m}}+\sigma_{g}^{2} \mathbf{R}_{\mathbf{h}}^{-\mathbf{1}}\right]^{-1} \mathbf{S}_{\Delta \theta_{m}}^{H}\left(\mathbf{S}_{\Delta \theta_{m}} \mathbf{h}\right)+\mathbf{g}^{\prime},
$$

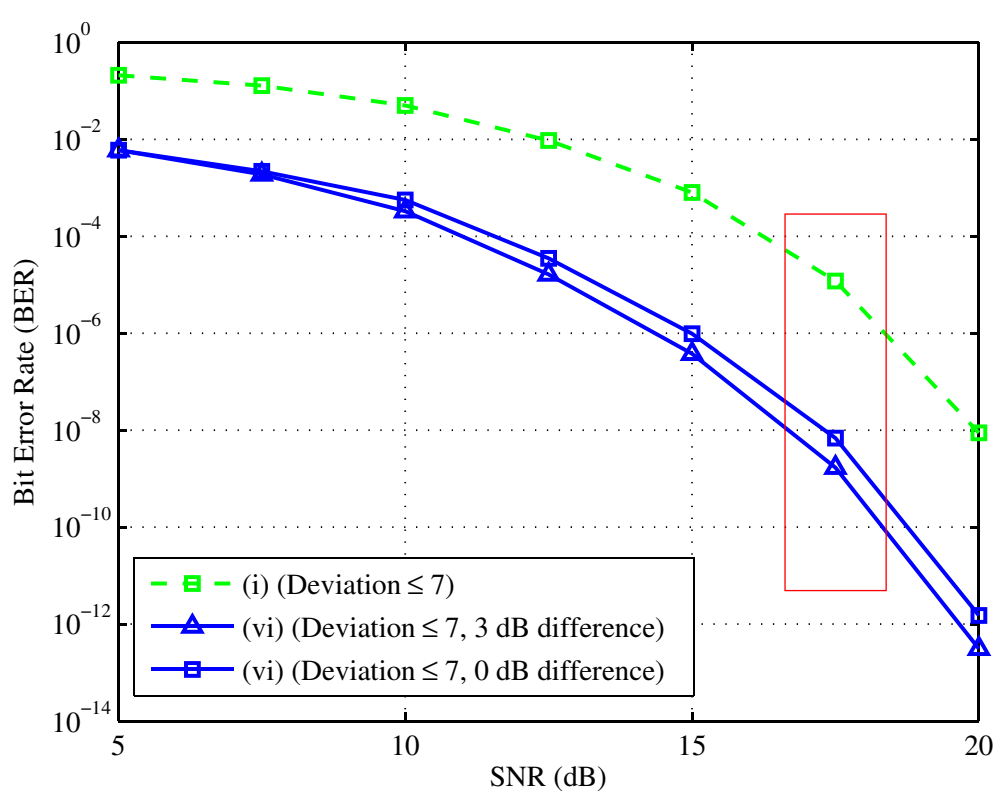

Figure 12 BER under BRAN-A channel. 
where $\mathbf{g}^{\prime}=\left[\mathbf{S}_{\Delta \theta_{m}}^{H} \mathbf{S}_{\Delta \theta_{m}}+\sigma_{g}^{2} \mathbf{R}_{\mathbf{h}}^{-\mathbf{1}}\right]^{-1} \mathbf{S}_{\Delta \theta_{m}}^{H} \boldsymbol{\Phi}_{\Delta \theta_{m}, \hat{\epsilon}_{m}}^{H} \mathbf{g . ~ N o t e}$ that in the case of perfect FS, from $\widehat{\mathbf{h}}_{\Delta \theta_{m}}=\left[\mathbf{S}_{\Delta \theta_{m}}^{H}\right.$

$$
\begin{aligned}
& \left.\mathbf{S}_{\Delta \theta_{m}}+\sigma_{g}^{2} \mathbf{R}_{\mathbf{h}}^{-\mathbf{1}}\right]^{-1} \mathbf{S}_{\Delta \theta_{m}}^{H} \mathbf{r}_{\Delta \theta_{m}} \text {, we obtain } \\
& \widehat{\mathbf{h}}_{\Delta \theta_{m}}=\left[\mathbf{S}_{\Delta \theta_{m}}^{H} \mathbf{S}_{\Delta \theta_{m}}+\sigma_{g}^{2} \mathbf{R}_{\mathbf{h}}^{-\mathbf{1}}\right]^{-1} \mathbf{S}_{\Delta \theta_{m}}^{H}\left(\mathbf{S}_{\Delta \theta_{m}} \mathbf{h}\right)+\mathbf{g}^{\prime \prime},
\end{aligned}
$$

where $\mathbf{g}^{\prime \prime}=\left[\mathbf{S}_{\Delta \theta_{m}}^{H} \mathbf{S}_{\Delta \theta_{m}}+\sigma_{g}^{2} \mathbf{R}_{\mathbf{h}}^{-\mathbf{1}}\right]^{-1} \mathbf{S}_{\Delta \theta_{m}}^{H} \mathbf{g}$. Thus, we prove that Equations 46 and 47 are similar and thus reflects the fact that the PSF curves are approximately the same with or without CFO perfect knowledge.

We addressed the question of whether the estimated transmission channel $\widehat{\mathbf{h}}$ (see Section 4.2.1) during the transmission medium negotiation may change when DATA is allowed to be transmitted especially when stations move. If the $\mathrm{CtS}$ control frame has been correctly received, the interval time $\Delta t$ (see Figure 4 ) given by the difference between the starting time of the transmitted DATA frame and the RtS control frame is in the worst case equal to $124 \mu$ s (i.e., $T_{\mathrm{RtS}}+T_{\mathrm{CtS}}+2 T_{\mathrm{SIFS}}$ ) when the rate is set to $6 \mathrm{Mb} / \mathrm{s}$ [26]. With a walking speed of $1.5 \mathrm{~m} / \mathrm{s}$ and a carrier frequency of $5.2 \mathrm{GHz}$, the maximum Doppler frequency $f_{D}$ is equal to $26 \mathrm{~Hz}$. Each $j$ th channel tap is multiplied by $e^{j 2 \pi f_{D} \Delta t} \approx e^{j 0.02}$ and therefore does not effect the estimated channel $\widehat{\mathbf{h}}$. This is confirmed by the simulation results provided in Figures 9 and 10. The curves denoted by 'Joint MAP TS-SF (Doppler freq., deviation $\leq$ 4)' in Figure 9 and by 'Joint MAP TS-SF (Doppler freq., deviation $\leq 7$ )' in Figure 10 are close to that of the cases without Doppler frequency.

Figures 11 and 12 refer to BER versus SNRs. DATA frame is demodulated as follows: the synchronized data signals are transformed into received symbols in frequency domain using FFT. These received symbols combined with channel estimation obtained by the second stage allows to estimate the data symbols which are then led into the de-mapping block (BPSK demodulation in this case), de-interleaving block, followed by the conventional Viterbi decoder at $R=1 / 2$. The estimated data bits are then processed by the descrambler block. A perfect frequency-domain equalizer is considered to compensate the deviations. The BER curves given in Figures 11 and 12 show that our synchronization algorithm provides better results compared to [19] regardless the selected channel model.

Clearly the synchronization algorithm, described by Equations 5, 6, and 9, with which we compared the performance of our algorithm in terms of PSF is less expensive in terms of computational complexity. Nevertheless, the question of an efficient implementation must be considered to reduce the full computational complexity of our algorithm. Indeed, our first study shows that many simplifications in terms of arithmetic computational are feasible. Note for example in Equation 24, the matrix inversion is calculated on diagonal matrices; the parameter $N$ is a power of 2 then the IFFT can be efficiently computed using the radix- 2 FFT algorithm. Moreover, some terms are defined in several places such as in Equation 32 where the covariance matrix has already been computed in Equation 24. The inverse matrix in Equation 32 can be computed according to Strassen's matrix inversion algorithm. In Equation 34, the calculation of the first derivative are re-used for calculating the second derivative thus reducing the computational load. In the standard operating mode, it is also possible to reduce the number of Newton-Raphson iterations (to two iterations) and the value of $M$. Table 2 provides the arithmetic complexity evaluated for each stage. Furthermore, the receiver latency issue is one of point to consider. The first steps of the receiver $(\mathrm{RtS} / \mathrm{CtS})$ are exactly equivalent to the classical algorithm in terms of delay. Concerning the processing of DATA frame, we introduce an intrinsic delay because we are awaiting for the SIGNAL field before applying our algorithm. This corresponds to an intrinsic delay of $4 \mu s$, while the total allowed delay corresponding to SIFS is equivalent to $16 \mu \mathrm{s}$. Other differences between the

Table 2 Arithmetic complexity of the proposed algorithm

\begin{tabular}{llll}
\hline Stage & Equations & $\#$ additions & $\sharp$ multiplications \\
\hline Initialization & $(18),(19)$ & 7 & 3 \\
First & $(21)$ & $2 N L_{\text {STF }}-\frac{3}{2} N^{2}$ & $2 N L_{\text {STF }}-\frac{3}{2} N^{2}$ \\
& & $\quad+\frac{1}{2} N-L_{\text {STF }}-1$ & $+\frac{1}{2} N+L$ \\
& $(22)$ & $N \log N+2 N$ & $\frac{N}{2} \log N+11 N$ \\
& $(23)$ & & $N$
\end{tabular}


classical and our proposed algorithm are only linked to computational complexity, and the corresponding delay is heavily dependent on the architecture of the receiver. This architectural design problem is out of the scope of our paper.

\section{Conclusion}

Based on the advantages of the DA and NDA approaches, this paper developed a time and frequency synchronization algorithm of mobile stations in IEEE 802.11a wireless network. In addition to the information specifically dedicated to synchronization (such as training sequences), these algorithms make use of an additional source of information obtained by careful examination of the standard. In fact, under some conditions, the receiver is able to predict this information when the CSMA/CA mechanism is triggered. Moreover the exchanged control frame (RtS) combined to the bit-rate adaptation algorithm to the channel is exploited to estimate the transmission channel before the first stage of the proposed algorithm. The first CTS stage exploits the identified SIGNAL field and an estimate of the channel to improve the symbol timing estimation. The second stage performs a joint MAP time and frequency synchronization. Finally, in the third stage, a timing metric in the frequency domain taking into account the presence of channel estimation errors is carried out. Even if the power level used to transmit RtS/CtS control frames is the same as that deployed to transmit DATA frames, simulation results show that the proposed synchronization algorithm achieves significant improvements measured in terms of PSF and BER, in both indoor and outdoor environments.

\section{Competing interests}

The authors declare that they have no competing interests.

\section{Acknowledgements}

This work was supported by the Ministry of Science and Technology of Vietnam, under project number 39/2012/HD/NDT.

\section{Author details}

${ }^{1}$ L2TI, Institut Galilée, Université Paris 13, Sorbonne Paris Cité, 99 avenue Jean-Baptiste Clément, F93430 Villetaneuse, France. ${ }^{2}$ LSS/CNRS, Supélec, 3 rue Joliot Curie, F91192 Gif sur Yvette, France. ${ }^{3}$ VNU University of Engineering and Technology, 144 Xuan Thuy, Cau Giay, Hanoi, Vietnam.

Received: 12 March 2014 Accepted: 3 February 2015

Published online: 26 February 2015

\section{References}

1. IEEE Std.802.11a (1999)

2. T Pollet, MV Bladel, M Moeneclaey, BER sensitivity of OFDM systems to carrier frequency offset and Wiener phase noise. IEEE Trans. Commun. 43(2/3/4), 887-895 (1995). doi:10.1109/26.380034

3. SR Herlekar, C Zhang, HC Wu, A Srivastava, Y Wu, OFDM performance analysis in the phase noise arising from the hot-carrier effect. IEEE Trans. Consumer Electron. 52(3), 757-765 (2006). doi:10.1109/TCE.2006.1706467

4. M Speth, F Classen, H Meyr, in IEEE 47th Vehicular Technlogy Conference (VTC, Phoenix, AZ), vol. 3. Frame synchronization of OFDM systems in frequency selective fading channels, (1997), pp. 1807-1811. doi:10.1109/VETEC.1997.605870

5. JJ van de Beek, M Sandell, M Isaksson, PO Borjesson, in Proc. IEEE Int. Conf. Universal Personal Commun. Low complex frame synchronization in OFDM systems, (1995), pp. 982-986. doi:10.1109/ICUPC.1995.497156

6. Ph J Tourtier, R Monnier, P Lopez, Multicarrier modem for digital HDTV terrestrial broadcasting. Signal Process.: Image Commun. 5(5-6), 379-403 (1993). doi:10.1016/0923-5965(93)90004-D

7. YS Cho, J Kim, WY Yang, CG Kang, MIMO-OFDM Wireless Communications with MATLAB. (John Wiley \& Sons, 2010)

8. N Benvenuto, S Tomasin, On the comparison between OFDM and single carrier modulation with a DFE using a frequency-domain feedforward filter. IEEE Trans. Commun. 50(6), 947-955 (2002). doi:10.1109/TCOMM.2002.1010614

9. S Ma, X Pan, GH Yang, TS Ng, Blind symbol synchronization based on cyclic prefix for OFDM systems. IEEE Trans. Vehicular Technol. 58(4), 1746-1751 (2009). doi:10.1109/TVT.2008.2004031

10. B Park, E Ko, H Cheon, C Kang, D Hong, in Proc. IEEE Global Telecom. Conf. vol. 5. A blind OFDM synchronization algorithm based on Cyclic correlation, (2001), pp. 3116-3119. doi:10.1109/GLOCOM.2001.966000

11. JJ Van de Beek, M Sandell, PO Borjesson, ML estimation of time and frequency offset in OFDM systems. IEEE Trans. Signal Process. 45(7), 1800-1805 (1997). doi:10.1109/78.599949

12. M Sandell, Beek Van de JJ, PO Borjesson, in Proc. Int. Symp Synchronization. Timing and frequency synchronization in OFDM systems using the cyclic prefix, (1995), pp. 16-19

13. AJ Al-Dweik, A non-data-aided symbol timing recovery technique for OFDM systems. IEEE Trans. Commun. 54(1), 37-40 (2006). doi:10.1109/TCOMM.2005.861676

14. TM Schmidl, DC Cox, Robust frequency and timing synchronization for OFDM. IEEE Trans. Commun. 45(12), 1613-1621 (1997). doi:10.1109/26.650240

15. BY Prasetyo, F Said, AH Aghvami, Fast burst synchronisation technique for OFDM-WLAN systems. in IEEE Proc. Commun. 147(5), 292-298 (2000) doi:10.1049/ip-com:20000612

16. H Nguyen-Le, T Le-Ngoc, Pilot-aided joint CFO doubly-selective channel estimation for OFDM transmissions. IEEE Trans. Broadcasting. 56(4), 514-522 (2010). doi:10.1109/TBC.2010.2055673

17. HT Hsieh, WR Wu, Maximum likelihood timing and carrier frequency offset estimation for OFDM systems with periodic preambles. IEEE Trans. Vehicular Technol. 58(8), 4224-4237 (2009). doi:10.1109/TVT.2009.2019820

18. SK Manusani, RS Kshetrimayum, R Bhattacharjee, in Indiana conference, Annual IEEE. Robust time and frequency synchronization in OFDM based 802.11a WLAN systems, (2006), pp. 1-4. doi:10.1109/INDCON. 2006.302775

19. MJ Canet, V Almenar, J Marin-Roig, J Valls, in Int. Symp. on Personal, Indoor and Mobile Radio Communications (PIMRC). Time synchronization for the IEEE 802.11a/g WLAN standard, (2007), pp. 1-5. doi:10.1109/PIMRC.2007.4394066

20. Y Zhang, J Zhang, M Xia, in IEEE Int. Vehicular Technology Conf. Joint timing synchronization and channel estimation for OFDM systems via MMSE criterion, (2008), pp. 1-4. doi:10.1109/NETECF.2008.239

21. CL Nguyen, A Mokraoui, P Duhamel, N Linh-Trung, in Proceedings of the 20th European Signal Processing Conference (EUSIPCO). Time synchronization algorithm in IEEE 802.11a communication system, (2012), pp. 1628-1632

22. CL Nguyen, A Mokraoui, P Duhamel, N Linh-Trung, in Int. Conference on Advanced Technologies for Communications (ATC). Enhanced time synchronization for IEEE 802.11a system using SIGNAL field and MAP channel estimation, (2012), pp. 46-49. doi:10.1109/ATC.2012.6404226

23. CL Nguyen, A Mokraoui, P Duhamel, N Linh-Trung, in IEEE Int. Conference on Acoustics, Speech and Signal Processing (ICASSP). Improved time synchronization in presence of imperfect channel state information, (2013), pp. 4903-4907. doi:10.1109/ICASSP.2013.6638593

24. CL Nguyen, A Mokraoui, P Duhamel, N Linh-Trung, Improved time and frequency synchronization algorithm for IEEE 802.11a wireless standard based on SIGNAL field. REV J. Electron. Comm. 3(1-2), 40-49 (2013)

25. FR Gfeller, W Hirt, U.S. Patent 6,643,469. Nov 2003

26. M Ergen, P Varaiya, Throughput analysis and admission control for IEEE 802.11a. Mobile Networks and App. 10, 705-716 (2005) 
27. Z Li, A Das, AK Gupta, S Nandi, Full auto rate MAC protocol for wireless ad-hoc networks. IEE Proc. Commun. 152(3), 311-319 (2005). doi:10.1049/ip-com:20041022

28. M Gast, 802.11 Wireless Networks: The Definitive Guide, 2nd edn. (O'Reilly Media, 2005)

29. P Piantanida, S Sadough, P Duhamel, On the outage capacity of a practical decoder accounting for channel estimation inaccuracies. IEEE Trans. Commun. 57(5), 1341-1350 (2009). doi:10.1109/TCOMM.2009.070353

30. M Pätzold, Mobile fading channels, 2nd edn. (John Wiley and Sons, 2002)

31. ETSI Normalization Committee: Channel models for HIPERLAN/2 in different indoor scenarios (1998). http://www.etsi.org.

32. Y Mostofi, DC Cox, Mathematical analysis of the impact of timing synchronization errors on the performance of an OFDM system. IEEE Trans. Commun. 54(2), 226-230 (2006). doi:10.1109/TCOMM.2005.861675

33. F Peng, WE Ryan, in IEEE Wireless Communications and Networking Conference (WCNC, Las Vegas, NV). Low-complexity soft demapper for OFDM fading channels with ICl, (2006), pp. 1549-1554. doi:10.1109/WCNC.2006.1696518

\section{Submit your manuscript to a SpringerOpen ${ }^{\circ}$ journal and benefit from:}

- Convenient online submission

- Rigorous peer review

- Immediate publication on acceptance

- Open access: articles freely available online

- High visibility within the field

- Retaining the copyright to your article 